\title{
Synthesis of a Human Urinary Metabolite of Prostaglandin $D_{2}$
}

\author{
Jennifer R. Kimbrough ${ }^{\omega}$, Zachary Austin ${ }^{\omega}$, Ginger L. Milne ${ }^{\xi, \phi} \&$ Gary A. Sulikowski ${ }^{\omega}, \xi, \phi^{*}$ \\ wDepartment of Chemistry, Vanderbilt University, Nashville, TN 37232, USA \\ ¿Department of Pharmacology, Vanderbilt University, Nashville, TN 37232, USA \\ $\phi$ Vanderbilt Institute of Chemical Biology, Vanderbilt University, Nashville, TN 37232, USA
}

\section{Supporting Information}

1. General Procedures

2. Materials

3. Instrumentation

4. Compound Preparation

5. Copy of ${ }^{1} \mathrm{H}$ and ${ }^{13} \mathrm{C}$ NMR Spectra

6. References and footnotes
S2

S2

S2

S3-S11

S12-S40

S41 
1. General Procedure: All non-aqueous reactions were performed in flame-dried or oven dried roundbottomed flasks under an atmosphere of argon. Stainless steel syringes or cannula were used to transfer air- and moisture-sensitive liquids. Reaction temperatures were controlled using a thermocouple thermometer, analog hotplate stirrer equipped with an oil bath unless otherwise noted. Reactions were conducted at room temperature (approximately $23{ }^{\circ} \mathrm{C}$ ) unless otherwise noted. Flash column chromatography was conducted using silica gel 230-400 mesh. Reactions were monitored by analytical thin-layer chromatography, using EMD Silica Gel 60 F254 glass-backed pre-coated silica gel plates. The plates were visualized with UV light $(254 \mathrm{~nm})$ and stained with potassium permanganate or $p$ anisaldehyde-sulfuric acid followed by charring. Yields were reported as isolated, spectroscopically pure compounds.

2. Materials: Solvents and chemicals were purchased from Sigma-Aldrich. Dry dichloromethane $\left(\mathrm{CH}_{2} \mathrm{Cl}_{2}\right)$ was collected from an MBraun MB-SPS solvent system. Triethylamine, N,N-dimethylformamide (DMF) and dimethyl sulfoxide (DMSO) were used as received in a bottle with a Sure/Seal. N,N-diisopropylethylamine was distilled from calcium hydride and stored over $\mathrm{KOH}$. Deuterated solvents were purchased from Cambridge Isotope Laboratories. All commercial reagents were used as received. Racemic 4-(tertbutyldimethylsilyloxy)cyclopent-2-en-1-one (3) was prepared according to the procedures of Deardorff ${ }^{1}$ and Tietze ${ }^{2}$.

3. Instrumentation: ${ }^{1} \mathrm{H}$ NMR spectra were recorded on Bruker 400 or $600 \mathrm{MHz}$ spectrometers and are reported relative to internal chloroform $\left({ }^{1} \mathrm{H}, \delta 7.26\right)$, methanol $\left({ }^{1} \mathrm{H}, \delta 3.31\right)$, and DMSO $\left({ }^{1} \mathrm{H}, \delta 2.50\right)$. Data for ${ }^{1} \mathrm{H}$ NMR spectra are reported as follows: chemical shift $(\delta \mathrm{ppm})$, multiplicity $(\mathrm{s}=$ singlet, $\mathrm{d}=\mathrm{doublet}$, $\mathrm{t}=$ triplet, $\mathrm{q}=$ quartet, $\mathrm{m}=$ multiplet, $\mathrm{br}=$ broad), coupling constants $(\mathrm{Hz})$, and integration. In the case of isomeric mixtures, NMR signals corresponding to major and minor isomers are indicated (cmpds 7 and 15). For ${ }^{13} \mathrm{C}$ NMR, chemical shifts are bolded where both isomers resonate at identical frequency. ${ }^{13} \mathrm{C}$ NMR were recorded on Bruker 100 or $150 \mathrm{MHz}$ spectrometers and are reported relative to internal chloroform $\left({ }^{13} \mathrm{C}, \delta\right.$ 77.1), methanol $\left({ }^{13} \mathrm{C}, \delta 49.2\right)$, and DMSO $\left({ }^{13} \mathrm{C}, \delta 40.3\right)$. Noted, trace impurities were assigned in agreement with Gottlieb and Nudelman'. Low-resolution mass spectra were acquired on an Agilent Technologies Series 1200 single quad ChemStation autosampler system using electrospray ionization (ESI) in positive mode. High-resolution mass spectra (HRMS) were obtained from the Department of Chemistry and Biochemistry, University of Notre Dame Mass Spectrometry Center or the Mass Spectrometry Research Center at Vanderbilt University. 


\section{Compound Preparation}

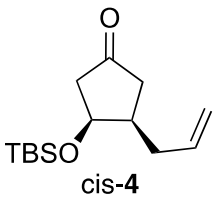

cis-4 To a solution of 4-(tert-butyldimethylsilyloxy)cyclopent-2-en-1-one (3) $(500 \mathrm{mg}$, $2.36 \mathrm{mmol})$ in $\mathrm{CH}_{2} \mathrm{Cl}_{2}(20 \mathrm{~mL})$ cooled to $-78{ }^{\circ} \mathrm{C}$ was added a solution of $\mathrm{TiCl}_{4}(2.36 \mathrm{~mL}$ of $1 \mathrm{M}$ solution in $\left.\mathrm{CH}_{2} \mathrm{Cl}_{2}, 2.36 \mathrm{mmol}\right)$. After $5 \mathrm{~min}$, allyl trimethylsilane $(0.515 \mathrm{~mL}, 2.06$ mmol) in $\mathrm{CH}_{2} \mathrm{Cl}_{2}(2.5 \mathrm{~mL})$ was added dropwise. The dark purple mixture stirred at -78 ${ }^{\circ} \mathrm{C}$ for $2 \mathrm{~h}$, at which point it was quenched with saturated aq $\mathrm{NaHCO}_{3}(15 \mathrm{~mL})$ and allowed to warm to room temperature. The aqueous layer was extracted with $\mathrm{Et}_{2} \mathrm{O}(3 \times 15 \mathrm{~mL})$ and combined organic extracts washed with brine $(15 \mathrm{~mL})$, dried $\left(\mathrm{MgSO}_{4}\right)$, filtered and concentrated in vacuo. The resultant residue was purified by flash column chromatography (silica gel, gradient elution, 10 to $40 \%$ EtOAc in hexanes) to afford $305 \mathrm{mg} \mathrm{(51 \% )}$ of cyclopentanone cis-4 as a colorless oil: ${ }^{1} \mathrm{H}$ NMR $\left(400 \mathrm{MHz} \mathrm{CDCl}_{3}\right) \delta 5.85-5.75(\mathrm{~m}$, 1H), 5.09-5.00 (m, 2H), 4.42 (br s, 1H), 2.37-2.09 (m, 7H), $0.86(\mathrm{~s}, 9 \mathrm{H}), 0.06(\mathrm{~s}, 3 \mathrm{H}), 0.04(\mathrm{~s}, 3 \mathrm{H}) ;{ }^{13} \mathrm{C} \mathrm{NMR}$ $\left(100 \mathrm{MHz}_{\mathrm{C}} \mathrm{CDCl}_{3}\right) \delta 217.4,136.8,116.1,71.5,49.4,43.1,41.0,34.2,25.9,18.1,-4.4,-4.8$. Compound characterization data was consistent with previous report. ${ }^{3}$

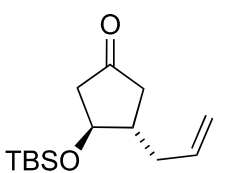

trans-4

trans -4 To a stirred mixture of $\mathrm{CuBr} \cdot \mathrm{SMe}_{2}(162 \mathrm{mg}, 0.790 \mathrm{mmol})$ and $\mathrm{LiCl}(33 \mathrm{mg}, 0.768$ $\mathrm{mmol})$ in THF $(1.5 \mathrm{~mL})$ at $-78{ }^{\circ} \mathrm{C}$ was added allyl magnesiumbromide $(0.707 \mathrm{~mL}$ of $1.0 \mathrm{M}$ in $\left.\mathrm{Et}_{2} \mathrm{O}, 0.707 \mathrm{mmol}\right), \mathrm{TMSCl}(97 \mu \mathrm{L}, 0.784 \mathrm{mmol})$ followed by a solution of 4 -(tertbutyldimethylsilyloxy)cyclopent-2-en-1-one (3) $(75 \mathrm{mg}, 0.354 \mathrm{mmol})$ in THF $(1.0 \mathrm{~mL})$.

The solution was stirred for $5 \mathrm{~min}$ at $-78{ }^{\circ} \mathrm{C}$, at which point it was quenched with a 1:9 solution of saturated aq $\mathrm{NH}_{4} \mathrm{Cl}(3 \mathrm{~mL})$. The mixture was extracted with EtOAc $(3 \times 5 \mathrm{~mL})$ and the combined organic layers were dried (MgSO4), filtered and concentrated in vacuo. The resultant residue was purified by flash chromatography (silica gel, 10\% EtOAc in hexanes) to yield $18 \mathrm{mg}(20 \%)$ of the cyclopentanone trans-4 as a clear oil: ${ }^{1} \mathrm{H}$ NMR $\left(400 \mathrm{MHz}, \mathrm{CDCl}_{3}\right) \delta$ 5.82-5.72 $(\mathrm{m}, 1 \mathrm{H}), 5.08-5.03(\mathrm{~m}, 2 \mathrm{H}), 4.13-4.08(\mathrm{~m}, 1 \mathrm{H})$, 2.55-2.47 (m, 2H), 2.38-2.31 (m, 1H), 2.27-2.14 (m, 2H), 2.02-1.95 (m, 1H), $1.89(\mathrm{dd}, J=18.6,7.5 \mathrm{~Hz}, 1 \mathrm{H})$, 0.88 (s, 9H), 0.08 (s, 3H), 0.05 (s, 3H); ${ }^{3} \mathrm{C}$ NMR (150 MHz, $\left.\mathrm{CDCl}_{3}\right) \delta$ 216.3, 135.9, 117.0, 74.0, 47.4, 44.9, $42.3,36.9,25.9,18.1,-4.5,-4.7$. Compound characterization data was consistent with previous report. ${ }^{4}$

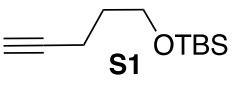

S1 To a solution of pentynol $(6.00 \mathrm{~g}, 71.3 \mathrm{mmol})$ in DMF $(72 \mathrm{~mL})$ was added TBSCl

(13.98 g, $92.7 \mathrm{mmol}), \mathrm{ImH}(9.70 \mathrm{~g}, 43 \mathrm{mmol})$ and DMAP (871 $\mathrm{mg}, 7.13 \mathrm{mmol})$. The solution was stirred overnight and quenched with water $(70 \mathrm{~mL})$, extracted with $\mathrm{Et}_{2} \mathrm{O}(4 \times 60 \mathrm{~mL})$. The combined organic extracts were washed with water $(50 \mathrm{~mL})$, brine $(50 \mathrm{~mL})$, dried $\left(\mathrm{MgSO}_{4}\right)$, filtered and 
concentrated in vacuo. The resultant residue was purified by flash column chromatography (silica gel, gradient elution, $0-20 \%$ EtOAc in hexanes) to yield $13.92 \mathrm{~g}(>95 \%)$ of the alkyne as a colorless oil. Spectral data matched reported literature values. ${ }^{5}$

S2 To a solution of the alkyne $\mathbf{S 1}(5.93 \mathrm{~g}, 29.9 \mathrm{mmol})$ in THF $(75 \mathrm{~mL})$ at $-78{ }^{\circ} \mathrm{C}$ was added $n$-BuLi ( $23.0 \mathrm{~mL}$ of $2.21 \mathrm{M}$ in hexanes, $50.9 \mathrm{mmol}$ ) dropwise over $5 \mathrm{~min}$. The solution stirred at $-78{ }^{\circ} \mathrm{C}$ for $1 \mathrm{~h}$, before paraformaldehyde $4.39(3.15 \mathrm{~g}, 0.11 \mathrm{~mol})$ was added and the reaction slowly warmed to room temperature over $3.5 \mathrm{~h}$. The mixture was quenched with saturated aq $\mathrm{NH}_{4} \mathrm{Cl}$ solution $(100 \mathrm{~mL})$, extracted with EtOAc $(3 \times 80 \mathrm{~mL})$. The combined organic extracts were dried $\left(\mathrm{MgSO}_{4}\right)$, filtered and concentrated in vacuo. The resultant residue was purified by flash column chromatography (silica gel, gradient elution, 5-40\% EtOAc in hexanes) to yield $6.07 \mathrm{~g}(89 \%)$ of propargyl alcohol $\mathbf{S} 2$ as a colorless oil. Spectral data matched reported literature values. ${ }^{6}$

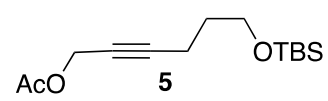

6-(tert-butyldimethylsilyloxy)hex-2-yn-1-yl acetate (5) To a solution of the propargyl alcohol $\mathbf{S 2}(6.04 \mathrm{~g}, 26.5 \mathrm{mmol})$ in pyridine $(26.5 \mathrm{~mL})$ was added $\mathrm{Ac}_{2} \mathrm{O}$ $(5.0 \mathrm{~mL})$. The reaction stirred overnight before it was diluted in EtOAc $(100 \mathrm{~mL})$, washed with sat. aq. $\mathrm{CuSO}_{4}(3 \times 40 \mathrm{~mL})$ and saturated aq $\mathrm{NaHCO}_{3}(40 \mathrm{~mL})$. The organic extract was dried $\left(\mathrm{MgSO}_{4}\right)$, filtered and concentrated in vacuo. The resultant residue was purified by flash column chromatography (silica gel, gradient elution, $0-10 \% \mathrm{Et}_{2} \mathrm{O}$ in hexanes) to yield $5.35 \mathrm{~g}(74 \%)$ of propargyl acetate 5 as a colorless oil: ${ }^{1} \mathrm{H}$ $\operatorname{NMR}\left(600 \mathrm{MHz}, \mathrm{CDCl}_{3}\right) \delta 4.66(\mathrm{t}, J=2.0 \mathrm{~Hz}, 2 \mathrm{H}), 3.68(\mathrm{t}, J=6.0 \mathrm{~Hz}, 2 \mathrm{H}), 2.31(\mathrm{tt}, J=7.1,2.1 \mathrm{~Hz}, 2 \mathrm{H})$, $2.09(\mathrm{~s}, 3 \mathrm{H}), 1.71(\mathrm{~m}, J=6.6 \mathrm{~Hz}, 2 \mathrm{H}), 0.89(\mathrm{~s}, 9 \mathrm{H}), 0.05(\mathrm{~s}, 6 \mathrm{H}) ;{ }^{33} \mathrm{C} \mathrm{NMR}\left(150 \mathrm{MHz}, \mathrm{CDCl}_{3}\right) \delta$ 170.5, 87.4, 74.2, 61.6, 53.0, 31.5, 26.1, 21.0, 18.5, 15.4, -5.2; HRMS (ESI) calc'd for $\mathrm{C}_{14} \mathrm{H}_{27} \mathrm{O}_{3} \mathrm{Si}[\mathrm{M}+\mathrm{H}]+:$ : 271.1729; found: 271.1724 .

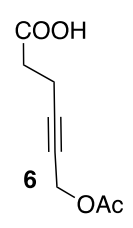

6-acetoxyhex-4-ynoic acid (6) Jones reagent was prepared by dissolving chromium trioxide $(5.93 \mathrm{~g}, 59.3 \mathrm{mmol})$ in water $(19 \mathrm{~mL})$ followed by the addition of concentrated sulfuric acid $(5.90 \mathrm{~mL})$ at $0{ }^{\circ} \mathrm{C}$. To the Jones reagent at $0{ }^{\circ} \mathrm{C}$ was added a solution of acetate $5(5.25 \mathrm{~g}$, $19.8 \mathrm{mmol}$ ) in acetone $(85 \mathrm{~mL})$ dropwise via an addition funnel over $15 \mathrm{~min}$. The ice bath was then removed and the reaction stirred at room temperature for $3 \mathrm{~h}$. Isopropanol $(90 \mathrm{~mL})$ was then added and the mixture was filtered through a plug of Celite and partially concentrated in vacuo. The filtrate was then dissolved in EtOAc $(50 \mathrm{~mL})$. washed with sat. aq. $\mathrm{NH}_{4} \mathrm{Cl}(2 \times 50 \mathrm{~mL})$ and the organic layer dried (MgSO4), filtered and concentrated in vacuo. The resultant residue was purified by flash column chromatography (silica gel, gradient elution, 25-50\% EtOAc in hexanes) to yield $2.30 \mathrm{~g}(68 \%)$ of carboxylic acid 6 as a white solid: ${ }^{1} \mathrm{H}$ NMR $\left(600 \mathrm{MHz}, \mathrm{CDCl}_{3}\right) \delta 4.65(\mathrm{t}, \mathrm{J}=2.1 \mathrm{~Hz}, 2 \mathrm{H})$ 2.58-2.63 (m, 2H), 2.53-2.58 
(m, 2H), 2.09 (s, 3H); $\left.{ }^{13} \mathrm{C} \mathrm{NMR} \mathrm{(125} \mathrm{MHz,} \mathrm{CDCl}_{3}\right) \delta$ 176.9, 170.5, 85.2, 75.1, 52.7, 33.0, 20.9, 14.6; HRMS (ESI) calc'd for $\mathrm{C}_{8} \mathrm{H}_{10} \mathrm{NaO}_{4}[\mathrm{M}+\mathrm{Na}]+:$ 193.0477; found: 193.0471 .

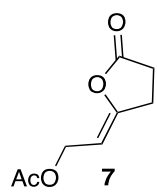

Allylic acetate 7 To a solution of the propargyl acetate $6(1.27 \mathrm{~g}, 7.47 \mathrm{mmol})$ in benzene (35 $\mathrm{mL}$ ) was added $\mathrm{Ag}_{2} \mathrm{CO}_{3}(206 \mathrm{mg}, 0.747 \mathrm{mmol})$. The solution was heated to reflux and stirred for

$8 \mathrm{~h}$. The mixture was then cooled to room temperature, filtered through a plug of Celite and concentrated in vacuo before the residue was purified by flash chromatography (silica gel, 0 to 50\% EtOAchexanes) to afford $1.14 \mathrm{~g}(90 \%)$ of allylic acetate 7 as $3: 1$ mixture of $Z: E$ isomers: ${ }^{1} \mathrm{H}$ NMR $(600 \mathrm{MHz}$, $\mathrm{CDCl}_{3}$ ) $\delta 5.39$ (tt, $1 \mathrm{H}, J=8.1,2.2 \mathrm{~Hz}, 1 \mathrm{H}$, minor), 4.87 (dd, $J=7.5,1.6 \mathrm{~Hz}, 1 \mathrm{H}$, major); 4.70 (d, $J=7.5 \mathrm{~Hz}$, $2 \mathrm{H}$, major), 4.56 (d, J= $8.5 \mathrm{~Hz}, 2 \mathrm{H}$, minor); 3.06-2.97 (m, 2H, minor), 2.94-2.85 (m, 2H, major); 2.75-2.64 (m, 2H); 2.05, (s, 3H); ${ }^{13} \mathrm{C}$ NMR (125 MHz, $\left.\mathrm{CDCl}_{3}\right) \delta 174.4$ (major), 174.1 (minor); 171.0; 155.1 (minor), 152.4 (major); 99.2; 59.4 (minor), 58.7 (major); 27.4; 25.1 (major), 22.9 (minor); 21.1; HRMS (ESI) calc'd for $\mathrm{C}_{8} \mathrm{H}_{10} \mathrm{NaO}_{4}[\mathrm{M}+\mathrm{Na}]+:$ 193.0477; found: 193.0471 .

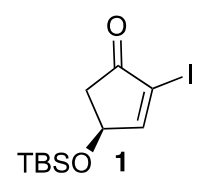

lodoenone 1 To a solution of cyclopentenone $3(2.04 \mathrm{~g}, 9.92 \mathrm{mmol})$ in $\mathrm{CH}_{2} \mathrm{Cl}_{2}(17.5 \mathrm{~mL})$ and pyridine $(17.5 \mathrm{~mL})$ at $0{ }^{\circ} \mathrm{C}$ was added a solution of iodine $(4.15 \mathrm{~g}, 16.3 \mathrm{mmol})$ in $\mathrm{CH}_{2} \mathrm{Cl}_{2}(17.5 \mathrm{~mL})$ and pyridine $(17.5 \mathrm{~mL})$ dropwise over $1 \mathrm{~h}$ via syringe pump. The mixture was then allowed to warm to room temperature and stirred for $30 \mathrm{~min}$ before $2 \mathrm{~N} \mathrm{HCl}(30$ $\mathrm{mL}$ ) was added slowly. The organic layer was washed with saturated aq $\mathrm{Na}_{2} \mathrm{SO}_{3}(2 \times 50 \mathrm{~mL})$, dried (MgSO4), filtered and concentrated in vacuo. The resultant residue was purified by flash chromatography (silica gel, gradient elution, $0-10 \%$ EtOAc in hexanes) to yield $2.99 \mathrm{~g}(92 \%)$ of iodocyclopentenone 1 as a pale-yellow solid: ${ }^{1} \mathrm{H}$ NMR $\left(400 \mathrm{MHz}, \mathrm{CDCl}_{3}\right) \delta 7.79(\mathrm{~d}, J=2.5 \mathrm{~Hz}, 1 \mathrm{H}), 4.96-4.94(\mathrm{~m}, 1 \mathrm{H}), 2.86(\mathrm{dd}, J=$ 18.2, $6.1 \mathrm{~Hz}, 1 \mathrm{H}$ ), 2.35 (dd, $J=18.2,2.0 \mathrm{~Hz}, 1 \mathrm{H}), 0.91(\mathrm{~s}, 9 \mathrm{H}), 0.14(\mathrm{~s}, 3 \mathrm{H}), 0.12(\mathrm{~s}, 3 \mathrm{H}) ;{ }^{13} \mathrm{C}$ NMR $(100$ $\left.\mathrm{MHz}, \mathrm{CDCl}_{3}\right) \delta 200.4,169.3,105.1,72.3,42.5,25.8,18.2,-4.58,-4.60$. Spectral data matched reported literature values. ${ }^{7}$

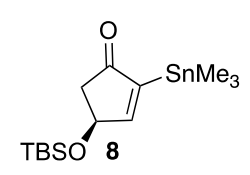

Trimethylstannylenone 8 To a solution of the iodoenone $1(342 \mathrm{mg}, 1.01 \mathrm{mmol})$ in benzene $(6.0 \mathrm{~mL})$ was added hexamethylditin $(0.42 \mathrm{~mL}, 2.02 \mathrm{mmol})$ and $\mathrm{Pd}\left(\mathrm{PPh}_{3}\right)_{4}(58$ $\mathrm{mg}, 0.05 \mathrm{mmol}$ ). Argon was passed through the solution for $10 \mathrm{~min}$, the solution was brought to reflux and maintained at reflux for $16 \mathrm{~h}$. The reaction mixture was then cooled to room temperature, diluted with pentanes $(10 \mathrm{~mL})$ and sat. aq. $\mathrm{KF}(7 \mathrm{~mL})$ was added. The tin salts were removed by filtration and the filtrate was washed with saturated aq KF $(10 \mathrm{~mL})$ and water $(2 \times 10 \mathrm{~mL})$, dried $\left(\mathrm{MgSO}_{4}\right)$, filtered and concentrated in vacuo. The resultant residue was purified by flash chromatography (silica gel, gradient elution, 5 to $10 \%$ ether in pentanes) to yield $305 \mathrm{mg}(80 \%)$ of stannenone 8 as a clear oil: ${ }^{1} \mathrm{H}$ NMR $\left(600 \mathrm{MHz}, \mathrm{CDCl}_{3}\right) \delta 7.50(\mathrm{~d}, J=2.2 \mathrm{~Hz}, 1 \mathrm{H}), 4.97(\mathrm{dt}, J=3.3,6.1 \mathrm{~Hz}, 1 \mathrm{H}), 2.71-2.65(\mathrm{dd}, J=$ 
17.9, $6.1 \mathrm{~Hz}, 1 \mathrm{H}), 2.24-2.19(\mathrm{dd}, J=18.0,2.6 \mathrm{~Hz}, 1 \mathrm{H}), 0.92(\mathrm{~s}, 9 \mathrm{H}), 0.25(\mathrm{~s}, 9 \mathrm{H}), 0.13(\mathrm{~s}, 3 \mathrm{H}), 0.12(\mathrm{~s}, 3 \mathrm{H})$; ${ }^{13} \mathrm{C} \mathrm{NMR}\left(150 \mathrm{MHz}, \mathrm{CDCl}_{3}\right) \delta 210.9,172.4,150.0,73.3,45.2,26.0,18.4,-4.50,4.53,-9.60$.

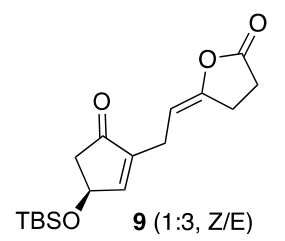

Cylopentenone 9 To a mixture of $\mathrm{LiCl}(361 \mathrm{mg}, 8.51 \mathrm{mmol})$ and $\mathrm{Pd}_{2}(\mathrm{dba})_{3}(389 \mathrm{mg}$, $0.425 \mathrm{mmol}$ ) was added the allylic acetate $6(434 \mathrm{mg}, 2.55 \mathrm{mmol}, 3: 1$ mixture of $Z: E$ isomers) in NMP (3.8 $\mathrm{mL})$. The resultant solution stirred for $15 \mathrm{~min}$ before trimethylstannylenone $8(800 \mathrm{mg}, 2.13 \mathrm{mmol})$ in NMP $(3.8 \mathrm{~mL})$ was added. Argon was bubbled through the reaction mixture for $15 \mathrm{~min}$ before it was heated to $50{ }^{\circ} \mathrm{C}$ and

stirred for $12 \mathrm{~h}$. The solution was then cooled to room temperature, diluted with ether $(50 \mathrm{~mL})$ and quenched by the addition of water $(30 \mathrm{~mL})$. The aqueous layer was extracted with ether $(2 \times 50 \mathrm{~mL})$ and EtOAc $(2 \times 50 \mathrm{~mL})$. The combined organic extracts were dried $\left(\mathrm{MgSO}_{4}\right)$, filtered and concentrated in vacuo. The resultant residue was purified by flash chromatography (silica gel, gradient elution, $5 \%$ to $100 \%$ ether in hexanes) to yield $647 \mathrm{mg}(94 \%)$ of enone 9 as a $3: 1$ mixture of $E$ and $Z$ isomers.

(E)-9 (major): $\mathrm{R}_{f} 0.29$ (4:1 hexanes: EtOAc); ${ }^{1} \mathrm{H}$ NMR $\left(600 \mathrm{MHz}, \mathrm{CDCl}_{3}\right) \delta 7.07$ (d, J= 2.2 Hz, $\left.1 \mathrm{H}\right), 5.25$ (tt, $J=8.0,2.2 \mathrm{~Hz}, 1 \mathrm{H}), 4.90(\mathrm{dt}, J=6.0,2.0 \mathrm{~Hz}, 1 \mathrm{H}), 2.90(\mathrm{t}, J=8.8 \mathrm{~Hz}, 2 \mathrm{H}), 2.83(\mathrm{~d}, J=8.0 \mathrm{~Hz}, 2 \mathrm{H}), 2.76$ (dd, $J=18.2,6.0 \mathrm{~Hz}, 1 \mathrm{H}), 2.67-2.71(\mathrm{~m}, 2 \mathrm{H}), 2.29$ (dd, $J=18.3,2.0 \mathrm{~Hz}, 1 \mathrm{H}), 0.91(\mathrm{~s}, 9 \mathrm{H}), 0.13(\mathrm{~s}, 3 \mathrm{H})$, $0.12(\mathrm{~s}, 3 \mathrm{H}) ;{ }^{13} \mathrm{C}$ NMR $\left(150 \mathrm{MHz}, \mathrm{CDCl}_{3}\right) \delta 205.7,174.8,157.3,151.1,145.1,100.1,69.1,45.6,27.6,25.9$, 22.7, 21.4, 18.3, -4.55; HRMS (ESI) calc'd for $\mathrm{C}_{17} \mathrm{H}_{26} \mathrm{O}_{4} \mathrm{Si}[\mathrm{M}+\mathrm{H}]^{+}:$323.1673; found: 323.1654.

(Z)-9 (minor): $\mathrm{R}_{f} 0.36$ (4:1 hexanes: EtOAc); ${ }^{1} \mathrm{H}$ NMR $\left(600 \mathrm{MHz}, \mathrm{CDCl}_{3}\right) \delta 7.05(\mathrm{~s}, 1 \mathrm{H}), 4.87$ (tt, $J=5.8,2.0$ $\mathrm{Hz}, 1 \mathrm{H}), 4.72(\mathrm{t}, J=7.5 \mathrm{~Hz}, 1 \mathrm{H}), 3.01(\mathrm{~d}, J=7.6 \mathrm{~Hz}, 2 \mathrm{H}), 2.84(\mathrm{t}, J=8.8 \mathrm{~Hz}, 2 \mathrm{H}), 2.73(\mathrm{dd}, J=18.2,5.8$ $\mathrm{Hz}, 1 \mathrm{H}), 2.61-2.68(\mathrm{~m}, 2 \mathrm{H}), 2.26(\mathrm{dd}, J=18.2,1.9 \mathrm{~Hz}, 1 \mathrm{H}), 0.89(\mathrm{~s}, 9 \mathrm{H}), 0.11(\mathrm{~s}, 3 \mathrm{H}), 0.10(\mathrm{~s}, 3 \mathrm{H}) ;{ }^{13} \mathrm{C}$ NMR $\left(150 \mathrm{MHz}, \mathrm{CDCl}_{3}\right) \delta 205.6,174.8,157.3,149.5,145.3,100.1,69.1$, 45.6, 27.9, 25.9, 25.0, 21.2, 18.2, -4.58; HRMS (ESI) calc'd for $\mathrm{C}_{17} \mathrm{H}_{26} \mathrm{O}_{4} \mathrm{Si}[\mathrm{M}+\mathrm{H}]^{+}:$323.1673; found: 323.1644 .

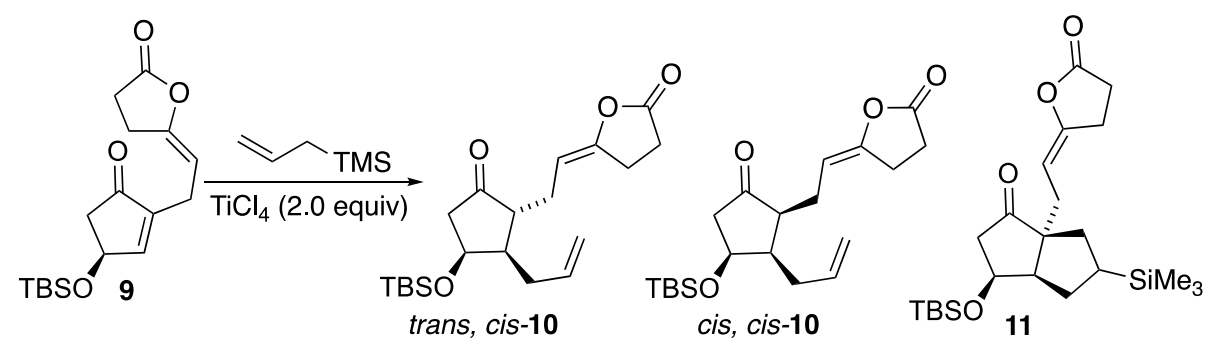

Allyl trimethylsilane addition to cyclopentenone (E)-9: To a solution of (E)-9 (272 mg, $0.844 \mathrm{mmol})$ dissolved in $\mathrm{CH}_{2} \mathrm{Cl}_{2}(7.0 \mathrm{~mL})$ at $-78{ }^{\circ} \mathrm{C}$ was added a solution of $\mathrm{TiCl}_{4}\left(850 \mu \mathrm{L}, 1 \mathrm{M}\right.$ solution in $\mathrm{CH}_{2} \mathrm{Cl}_{2}, 0.850$ $\mathrm{mmol})$ dropwise. A solution of allyltrimethylsilane $(403 \mu \mathrm{L}, 2.53 \mathrm{mmol})$ in $\mathrm{CH}_{2} \mathrm{Cl}_{2}$ was then added dropwise followed by a second addition of $\mathrm{TiCl}_{4}\left(850 \mu \mathrm{L}, 1 \mathrm{M}\right.$ solution in $\left.\mathrm{CH}_{2} \mathrm{Cl}_{2}, 0.850 \mathrm{mmol}\right)$. After stirring for $1.5 \mathrm{~h}$ at $-78{ }^{\circ} \mathrm{C}$, the reaction mixture was quenched by pouring into a solution of saturated aq $\mathrm{NaHCO}_{3}(25 \mathrm{~mL})$ 
and $\mathrm{CH}_{2} \mathrm{Cl}_{2}(25 \mathrm{~mL})$. The aqueous layer was extracted with $\mathrm{CH}_{2} \mathrm{Cl}_{2}(50 \mathrm{~mL})$, EtOAc $(50 \mathrm{~mL})$ and ether (50 $\mathrm{mL})$. The combined organic extracts were dried $\left(\mathrm{MgSO}_{4}\right)$, filtered and concentrated in vacuo. Purification by column chromatography (0-40\% EtOAc/hexanes) yielded trans,cis-10 as a clear oil $(112 \mathrm{mg}, 0.308 \mathrm{mmol}$, $36 \%$ ), cis, cis-10 as a clear oil (125 mg, $0.343 \mathrm{mmol}, 41 \%$ ), and silyl migration product 11 as a white solid (9 $\mathrm{mg}, 0.021 \mathrm{mmol}, 2 \%)$.

trans,cis-10: $\mathrm{R}_{f} 0.64$ (4:1 hexanes: EtOAc); ${ }^{1} \mathrm{H}$ NMR $\left(600 \mathrm{MHz}, \mathrm{CDCl}_{3}\right) \delta 5.80-5.94(\mathrm{~m}, 1 \mathrm{H}), 5.02-5.19(\mathrm{~m}$, $3 \mathrm{H}$ ), 4.41 (app t, $J=3.9 \mathrm{~Hz}, 1 \mathrm{H}$ ), 3.00-2.77 (m, 2H), 2.65 (app t, $J=8.6 \mathrm{~Hz}, 2 \mathrm{H}), 2.51-2.37(\mathrm{~m}, 2 \mathrm{H}), 2.36-$ $2.09(\mathrm{~m}, 5 \mathrm{H}), 2.00-1.90(\mathrm{~m}, 1 \mathrm{H}), 0.87(\mathrm{~s}, 9 \mathrm{H}), 0.07(\mathrm{~s}, 3 \mathrm{H}), 0.05(\mathrm{~s}, 3 \mathrm{H}) ;{ }^{13} \mathrm{C} \mathrm{NMR}\left(150 \mathrm{MHz}, \mathrm{CDCl}_{3}\right) \delta$ 218.4, 175.0, 151.0, 136.5, 116.5, 100.4, 69.3, 50.4, 48.9, 46.2, 32.9, 27.7, 25.9, 23.3, 22.8, 18.1, -4.24, 4.81; HRMS (ESI) calc'd for $\mathrm{C}_{20} \mathrm{H}_{33} \mathrm{O}_{4} \mathrm{Si}[\mathrm{M}+\mathrm{H}]+$ : 365.2143 ; found: 365.2161 .

cis,cis-10: $\mathrm{R}_{f} 0.57$ (4:1 hexanes: EtOAc); ${ }^{1} \mathrm{H}$ NMR $\left(600 \mathrm{MHz}, \mathrm{CDCl}_{3}\right) \delta 5.91-5.77(\mathrm{~m}, 1 \mathrm{H}), 5.30(\mathrm{app} \mathrm{tt}, J=$ 7.6, $2.2 \mathrm{~Hz}, 1 \mathrm{H}), 5.16-5.01(\mathrm{~m}, 2 \mathrm{H}), 4.44-4.40(\mathrm{~m}, 1 \mathrm{H}), 2.77(\mathrm{app} \mathrm{t}, J=8.0 \mathrm{~Hz}, 2 \mathrm{H}), 2.68-2.60(\mathrm{~m}, 2 \mathrm{H})$, $2.44(\mathrm{dd}, J=18.5,5.4 \mathrm{~Hz}, 1 \mathrm{H}), 2.40-2.18(7 \mathrm{H}), 0.89(\mathrm{~s}, 9 \mathrm{H}), 0.08(\mathrm{~s}, 3 \mathrm{H}), 0.06(\mathrm{~s}, 3 \mathrm{H}) ;{ }^{13} \mathrm{C} \mathrm{NMR}(150 \mathrm{MHz}$, $\left.\mathrm{CDCl}_{3}\right) \delta 218.2,175.0,149.5,136.8,116.4,103.3,71.5,49.8,48.3,45.6,30.1,27.8,25.9,24.0,22.8,18.1$, -4.37, -4.84; HRMS (ESI) calc'd for $\mathrm{C}_{20} \mathrm{H}_{33} \mathrm{O}_{4} \mathrm{Si}[\mathrm{M}+\mathrm{H}]+:$ 365.2143; found: 365.2141 .

Cyclopentanone 11: $\mathrm{R}_{f} 0.78$ (4.1 hexanes: EtOAc); ${ }^{1} \mathrm{H}$ NMR $\left(400 \mathrm{MHz}, \mathrm{CDCl}_{3}\right)$ 5.15-5.06 (m, $\left.1 \mathrm{H}\right), 4.44$ (app q, $J=7.5,1 \mathrm{H}), 2.88-2.74(\mathrm{~m}, 2 \mathrm{H}), 2.65(\operatorname{app~t}, J=8.4,2 \mathrm{H}), 2.60-2.45(\mathrm{~m}, 2 \mathrm{H}), 2.39-2.17(\mathrm{~m}, 3 \mathrm{H})$, 2.08-1.94 (m, 2H), 1.55-1.40 (m, 1H), 1.37-1.18 (m, 2H), 1.03-0.91 (m, 1H), $0.89(\mathrm{~s}, 9 \mathrm{H}), 0.07(\mathrm{~s}, 3 \mathrm{H}), 0.05$ (s, 3H), 0.05 (s, 9H), -0.05 (s, 6H); ${ }^{13} \mathrm{C}$ NMR $(100 \mathrm{MHz}, \mathrm{CDCl} 3) \delta 222.4,174.9,151.4,100.5,69.6,64.6$, $51.9,49.3,40.9,32.9,29.2,27.7,27.5,26.0,22.8,18.3,-2.96,-4.60,-4.71$.

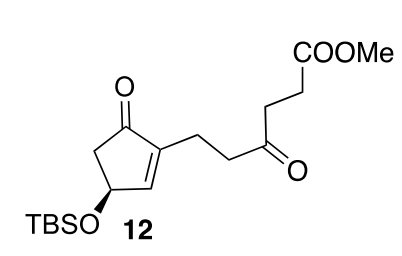

Methyl ester 12: To a stirred solution of enone $(E)-9$ (215 mg, 0.667 $\mathrm{mmol})$ dissolved in methanol was added a $0.2 \mathrm{~N}$ solution of $\mathrm{HCl}(0.10 \mathrm{~mL}$, $0.02 \mathrm{mmol})$. The reaction was stirred overnight and concentrated in vacuo. The crude product was dissolved in DMF $(2.5 \mathrm{~mL})$ and TBSCl (201 mg, $1.33 \mathrm{mmol})$, imidazole (136 mg, $2.00 \mathrm{mmol}$ ) and DMAP (8 mg, $0.067 \mathrm{mmol})$ added. The DMF solution was stirred overnight before and quenched with water $(2.0 \mathrm{~mL})$ and extracted with ether $(6 \times 10 \mathrm{~mL})$. The organic extracts were dried $\left(\mathrm{MgSO}_{4}\right)$, filtered and concentrated in vacuo. The resultant residue was purified by flash chromatography (silica gel, gradient elution, 5 to $15 \%$ EtOAc in hexanes) to yield $205 \mathrm{mg}(87 \%)$ of methyl ester 12 as a clear oil: ${ }^{1} \mathrm{HNMR}\left(400 \mathrm{MHz}, \mathrm{CDCl}_{3}\right) \delta$ $7.04(\operatorname{app~t}, J=1.17 \mathrm{~Hz}, 1 \mathrm{H}), 4.84(\mathrm{dd}, J=2.9,2.2 \mathrm{~Hz}, 1 \mathrm{H}), 3.63(\mathrm{~s}, 3 \mathrm{H}), 2.74-2.62(\mathrm{~m}, 5 \mathrm{H}), 2.56$ (app t, $J=$ $6.1 \mathrm{~Hz}, 2 \mathrm{H}$ ), 2.45 (app t, $J=7.2 \mathrm{~Hz}, 2 \mathrm{H}$ ), 2.22 (dd, $J=18.3,2.1 \mathrm{~Hz}, 1 \mathrm{H}$ ), 0.87 (s, 9H), 0.09 (s, 3H), 0.08 (s, $3 \mathrm{H}) ;{ }^{13} \mathrm{C}$ NMR $\left(100 \mathrm{MHz}, \mathrm{CDCl}_{3}\right) \delta 207.5,206.0,173.2,157.9,145.4,69.0,51.8,45.5,40.1,37.0,27.8$, $25.9,19.0,18.2,-4.63$. 


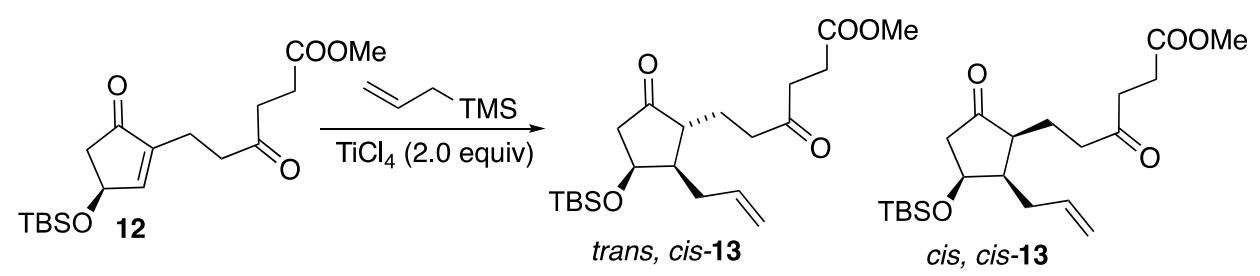

Allyl trimethylsilane addition to cyclopentenone 12: To a solution of the enone 12 (212 $\mathrm{mg}, 0.600$ mmol $)$ in $\mathrm{CH}_{2} \mathrm{Cl}_{2}(4.0 \mathrm{~mL})$ cooled to $-78{ }^{\circ} \mathrm{C}$ was added a solution of $\mathrm{TiCl}_{4}\left(600 \mu \mathrm{L}, 1.0 \mathrm{M}\right.$ in $\mathrm{CH}_{2} \mathrm{Cl}_{2}, 0.600$ $\mathrm{mmol})$, followed by a solution of allyltrimethylsilane $(285 \mu \mathrm{L}, 1.80 \mathrm{mmol})$ in $\mathrm{CH}_{2} \mathrm{Cl}_{2}(1.0 \mathrm{~mL})$ dropwise. After $1 \mathrm{~h}$, additional $\mathrm{TiCl}_{4}\left(600 \mu \mathrm{L}, 1.0 \mathrm{M}\right.$ in $\left.\mathrm{CH}_{2} \mathrm{Cl}_{2}, 0.600 \mathrm{mmol}\right)$ was added. The reaction continued to stir at $78^{\circ} \mathrm{C}$ for $1 \mathrm{~h}$ before quenched with sat. aq. $\mathrm{NaHCO}_{3}(3.0 \mathrm{~mL})$ and allowed to warm to room temperature. The mixture was diluted with $\mathrm{CH}_{2} \mathrm{Cl}_{2}(20 \mathrm{~mL})$, washed with saturated aq $\mathrm{NaHCO}_{3}(10 \mathrm{~mL})$, dried $\left(\mathrm{MgSO}_{4}\right)$, filtered and concentrated in vacuo. The resultant residue was purified by flash chromatography (silica gel, gradient elution, $5 \%$ to $20 \%$ EtOAc in hexanes) to yield $184 \mathrm{mg}(78 \%)$ of and inseparable $1: 1$ mixture of trans,cis- and cis, cis-13 as a clear oil.

Note: A pure sample of trans,cis-13 was obtained by acidic methanolysis of trans, cis-10 which enabled NMR assignment of the 1:1 mixture of trans, cis- and cis, cis-13.

cis,cis- and trans,cis-13: ${ }^{1} \mathrm{H}$ NMR $\left(400 \mathrm{MHz}, \mathrm{CDCl}_{3}\right) \delta 5.90-5.80(\mathrm{~m}, 1 \mathrm{H}), 5.11(\mathrm{dd}, J=17.2,1.6 \mathrm{~Hz}, 1 \mathrm{H})$, $5.04(\mathrm{~m}, 1 \mathrm{H}), 4.39(\mathrm{br} \mathrm{s}, 1 \mathrm{H}), 3.67(\mathrm{~s}, 3 \mathrm{H}), 2.82-2.49(\mathrm{~m}, 7 \mathrm{H}), 2.43-2.33(\mathrm{~m}, 1 \mathrm{H}), 2.29(\mathrm{~s}, 2 \mathrm{H}), 2.27-2.21(\mathrm{~m}$, $1 \mathrm{H}), 2.10-2.04(\mathrm{~m}, 1 \mathrm{H}), 1.97-1.80(\mathrm{~m}, 2 \mathrm{H}), 1.70-1.63(\mathrm{~m}, 1 \mathrm{H}), 0.86(\mathrm{~s}, 9 \mathrm{H}), 0.07(\mathrm{~s}, 3 \mathrm{H}), 0.04(\mathrm{~s}, 3 \mathrm{H})$; trans,cis-13 ${ }^{13} \mathrm{C}$ NMR $\left(100 \mathrm{MHz}, \mathrm{CDCl}_{3}\right) \delta 219.2,208.6,173.4,136.5,116.4,69.0,51.9,49.0,48.7,48.3$, 39.8, 37.2, 32.5, 29.9, 27.8, 25.9, 22.2, 18.1, -4.2, -4.9; cis, cis-13 ${ }^{13} \mathrm{C} \mathrm{NMR}\left(100 \mathrm{MHz}, \mathrm{CDCl}_{3}\right)$ 219.8, 208.6, $173.4,137.0,116.2,71.3,51.9,48.4,48.3,46.1,40.3,37.3,32.5,29.9,27.8,25.9,21.0,18.1,-4.4,-4.9$.

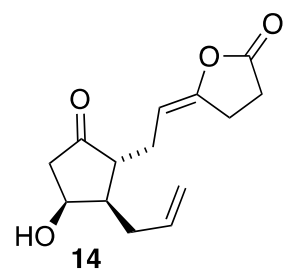

Alcohol 14: To a solution of trans, cis-10 (64 mg, $0.176 \mathrm{mmol})$ in $\mathrm{MeCN}(3.0 \mathrm{~mL})$ at 0 ${ }^{\circ} \mathrm{C}$ was added $70 \% \mathrm{HF}$-pyridine $(0.456 \mathrm{~mL}, 1.76 \mathrm{mmol}, 10$ equiv) dropwise. The reaction stirred at $0{ }^{\circ} \mathrm{C}$ for $1 \mathrm{~h}$ before additional $70 \% \mathrm{HF}$-pyridine $(0.456 \mathrm{~mL}, 1.76$ mmol, 10 equiv) was added. The reaction was stirred at $0{ }^{\circ} \mathrm{C}$ for $2 \mathrm{~h}$, and quenched with saturated aq $\mathrm{NaHCO}_{3}(50 \mathrm{~mL})$ followed by addition of $\mathrm{CHCl}_{3}(20 \mathrm{~mL})$. The product was extracted from the aqueous layer with $\mathrm{CHCl}_{3}(3 \times 20 \mathrm{~mL})$, organic layers were combined, dried $\left(\mathrm{MgSO}_{4}\right)$, filtered and concentrated in vacuo. The residue was purified by flash column chromatography (silica gel, gradient elution, 30-50\% EtOAc in hexanes) to yield $33 \mathrm{mg}(75 \%)$ of alcohol 14 as a colorless oil: ${ }^{1} \mathrm{H}$ NMR $\left(400 \mathrm{MHz}, \mathrm{CDCl}_{3}\right) \delta 5.98-5.80(\mathrm{~m}, 1 \mathrm{H}), 5.31-5.05(\mathrm{~m}, 3 \mathrm{H}), 4.67$ (app $\mathrm{t}, J=4.1 \mathrm{~Hz}, 1 \mathrm{H}), 2.98-2.76(\mathrm{~m}, 2 \mathrm{H}) ; 2.64(\mathrm{app} \mathrm{t}, J=8.51 \mathrm{~Hz}, 2 \mathrm{H}), 2.49-2.11(\mathrm{~m}, 6 \mathrm{H}), 2.06-1.96(\mathrm{~m}, 2 \mathrm{H})$; 
${ }^{13} \mathrm{C}$ NMR $\left(100 \mathrm{MHz}, \mathrm{CDCl}_{3}\right) \delta 218.1,175.0,151.1,136.1,117.1,100.3,68.5,50.2,48.0,45.1,33.1,27.7$, 23.2, 22.8 .

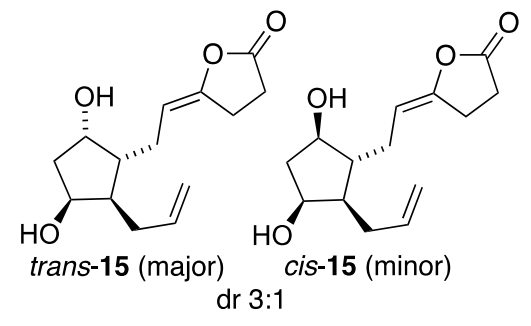

Diols trans- and cis-15: To a solution of ketone 14 (43 $\mathrm{mg}, 0.17 \mathrm{mmol})$ in THF $(2.0 \mathrm{~mL})$ was added tert-butylamine-borane complex $(67 \mathrm{mg}$, $0.77 \mathrm{mmol}, 4.5$ equiv). The solution was stirred at room temperature overnight before it was concentrated in vacuo. The resultant residue was purified by flash column chromatography (silica gel, gradient elution $5-30 \%$ EtOAc in hexanes) to yield $35 \mathrm{mg}(81 \%)$ of a $3: 1$ mixture of trans-(major) and cis-15 (minor) as a colorless oil: ${ }^{1} \mathrm{H}$ NMR $\left(400 \mathrm{MHz}, \mathrm{CDCl}_{3}\right) \delta 6.00-5.76(\mathrm{~m}, 1 \mathrm{H}) ; 5.32$ $5.21(\mathrm{~m}, 1 \mathrm{H}) ; 5.19-5.00(\mathrm{~m}, 2 \mathrm{H}) ; 4.39(\mathrm{~m}, 1 \mathrm{H}$, major), $4.32(\mathrm{~m}, 1 \mathrm{H}$, major) $4.22(\mathrm{~m}, 1 \mathrm{H}$, minor), $3.92(\mathrm{~m}$, $1 \mathrm{H}$, minor); 3.00- $2.74(\mathrm{~m}, 2 \mathrm{H}), 2.66$ (app t, $J=8.6 \mathrm{~Hz}, 2 \mathrm{H}), 2.42-1.72(\mathrm{~m}, 8 \mathrm{H}) ;{ }^{13} \mathrm{C} \mathrm{NMR}\left(100 \mathrm{MHz} \mathrm{CDCl}_{3}\right)$ $\delta 175.8$ (major), 174.9 (minor); 150.2 (minor), 149.7 (major); 138.0 (major), 137.6 (minor); 116.0; 103.4 (major), 102.3 (minor); 78.2 (minor), 75.0 (minor), 72.5 (major), 72.4 (major); 53.0 (minor), 50.1 (minor), 47.4 (major), 46.3 (major); 44.8 (major), 42.5 (minor); 33.9 (minor), 32.4 (major); 29.1 (minor), 27.84 (minor), 27.7 (major), 24.0 (major); 22.9 (minor), 22.8 (major).

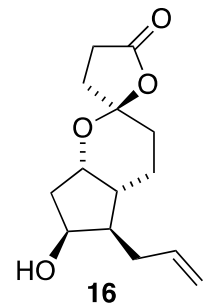

Spiroacetal 16: To a solution of a diol $15(33 \mathrm{mg}, 0.131 \mathrm{mmol})$ in $\mathrm{CH}_{2} \mathrm{Cl}_{2}(1.5 \mathrm{~mL})$ was added a solution of TFA (1.57mL, $0.5 \mathrm{M}$ in $\left.\mathrm{CH}_{2} \mathrm{Cl}_{2}, 0.24 \mathrm{mmol}\right)$. After $3 \mathrm{~h}$, additional TFA ( $0.48 \mathrm{~mL}, 0.5 \mathrm{M}$ in in $\mathrm{CH}_{2} \mathrm{Cl}_{2}, 0.24 \mathrm{mmol}$ ) was added before the reaction was quenched with excess triethylamine $(1.50 \mathrm{~mL})$, stirring continued for $1 \mathrm{~h}$ and concentrated in vacuo. Purification by flash column chromatography (silica gel, gradient elution $5-25 \%$ EtOAc in hexanes) yielded $26 \mathrm{mg}(79 \%)$ of a mixture of spiroacetal 16 as a colorless oil: ${ }^{1} \mathrm{H}$ NMR $\left(600 \mathrm{MHz}, \mathrm{CDCl}_{3}\right) \delta 5.96-5.89(\mathrm{~m}, 1 \mathrm{H}), 5.15(\mathrm{dd}, J=17.1,1.6 \mathrm{~Hz}, 1 \mathrm{H}), 5.07(\mathrm{dd}, J=10.2,0.9 \mathrm{~Hz}, 1 \mathrm{H})$, 4.40-4.38 (m, 2H), $2.76(\mathrm{dt}, J=17.7,9.9 \mathrm{~Hz}, 1 \mathrm{H}), 2.48$ (ddd, $J=17.7,9.5,2.5 \mathrm{~Hz}, 1 \mathrm{H}), 2.22-2.16(\mathrm{~m}, 4 \mathrm{H})$, 2.05-2.00 (m, 3H), 1.91-1.85 (m, 2H), 1.81-1.76 (m, 1H), 1.73-1.70 (m, 1H), 1.67-1.64 (m, 1H); ${ }^{13} \mathrm{C} \mathrm{NMR}$ $\left(150 \mathrm{MHz}, \mathrm{CDCl}_{3}\right) \delta 176.7,137.9,116.0,108.2,75.1,72.7,42.9,42.2,39.9,34.9,31.9,28.8,28.5,18.6$.

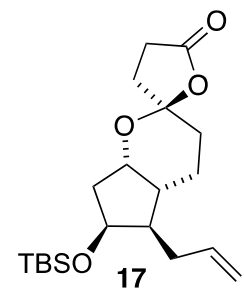

Spiroacetal 17: To a solution of alcohol $16(21 \mathrm{mg}, 0.083 \mathrm{mmol})$ in $\mathrm{CH}_{2} \mathrm{Cl}_{2}(1.0 \mathrm{~mL})$ at $0{ }^{\circ} \mathrm{C}$ was added 2,6-lutidine $(15 \mu \mathrm{L}, 0.125 \mathrm{mmol}, 1.5$ equiv) followed by TBSOTf (21 $\mu \mathrm{L}, 0.092 \mathrm{mmol}, 1.1$ equiv). The reaction was allowed to slowly warm to room temperature, stirred for $2 \mathrm{~h}$ and concentrated in vacuo. The resultant residue was purified by flash column chromatography (silica gel, gradient elution $5-10 \%$ EtOAc in hexanes) to afford $20 \mathrm{mg}(66 \%)$ of 17 as a colorless oil: ${ }^{1} \mathrm{H}$ NMR $\left(600 \mathrm{MHz}, \mathrm{CDCl}_{3}\right) \delta$

5.94-5.78 (m, 1H), 5.10- $4.91(\mathrm{~m}, 2 \mathrm{H}), 4.38-4.28(\mathrm{~m}, 2 \mathrm{H}), 2.85-2.68(\mathrm{~m}, 1 \mathrm{H}), 2.56-2.41(\mathrm{~m}, 1 \mathrm{H}), 2.42-1.38$ $(\mathrm{m}, 12 \mathrm{H}), 0.88(\mathrm{~s}, 9 \mathrm{H}), 0.03(\mathrm{~s}, 6 \mathrm{H}) ;{ }^{13} \mathrm{C} \mathrm{NMR}\left(150 \mathrm{MHz}, \mathrm{CDCl}_{3}\right) \delta 176.8,138.3,115.2,108.5,74.9,73.0$, 
44.6, 43.4, 40.1, 35.1, 32.0, 29.3, 28.6, 26.0, 19.3, 18.2, -4.1, -5.0; HRMS (ESI) calc'd for $\mathrm{C}_{20} \mathrm{H}_{35} \mathrm{O}_{4} \mathrm{Si}[\mathrm{M}+\mathrm{H}]+:$ 367.2299; found: 367.2289 .

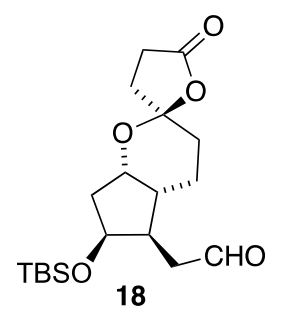

Aldehyde 18: Ozone was bubbled through a solution of spiroacetal 17 (24 mg, 0.065 $\mathrm{mmol})$ in $\mathrm{CH}_{2} \mathrm{Cl}_{2}(3.0 \mathrm{~mL})$ at $0{ }^{\circ} \mathrm{C}$ for ca. $3 \mathrm{~min}$ before triphenylphosphine $(172 \mathrm{mg}$, $0.656 \mathrm{mmol}$ ) was added. The solution was allowed to warm to room temperature, stirred overnight, and concentrated in vacuo. The resultant residue was purified by flash column chromatography (silica gel, gradient elution 5-40\% EtOAc in hexanes) to afford $20 \mathrm{mg}(83 \%)$ of 18 as a colorless oil: ${ }^{1} \mathrm{H}$ NMR $\left(600 \mathrm{MHz}, \mathrm{CDCl}_{3}\right) \delta 9.86(\mathrm{~s}, 1 \mathrm{H})$, $4.41(\mathrm{td}, J=6.3,3.4 \mathrm{~Hz}, 1 \mathrm{H}), 4.34(\mathrm{t}, J=4.8 \mathrm{~Hz}, 1 \mathrm{H}), 2.80-2.70(\mathrm{~m}, 2 \mathrm{H}), 2.56-2.44(\mathrm{~m}, 2 \mathrm{H}), 2.37(\mathrm{dd}, J=$ 18.0, 4.0 Hz, 1H), 2.20-2.13 (m, 2H), 2.07-1.97 (m, 2H), 1.86-1.78 (m, 3H), 1.72-1.68 (m, 1H), 1.57-1.51 $(\mathrm{m}, 1 \mathrm{H}), 0.86(\mathrm{~s}, 9 \mathrm{H}), 0.01(\mathrm{~s}, 3 \mathrm{H}),-0.02$ (s, 3H); ${ }^{13} \mathrm{C}$ NMR $\left(150 \mathrm{MHz}, \mathrm{CDCl}_{3}\right) \delta$ 202.5, 176.7, 108.1, 74.7, 72.7, 43.6, 42.7, 40.0, 38.3, 34.9, 28.6, 28.4, 25.9, 18.8, 18.0, -4.3, -5.0; HRMS (ESI) calc'd for $\mathrm{C}_{19} \mathrm{H}_{33} \mathrm{O}_{5} \mathrm{Si}[\mathrm{M}+\mathrm{H}]+:$ 369.2092; found: 369.2105 .

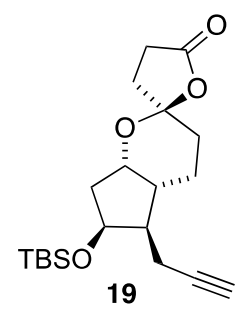

Alkyne 19: To a solution of Seyferth Gilbert reagent ${ }^{6}$ (48 $\mathrm{mg} 0.32 \mathrm{mmol}$ ) cooled to -78 ${ }^{\circ} \mathrm{C}$ in THF $(4.0 \mathrm{~mL})$ was added a solution of sodium bis(trimethylsilyl)amide $(0.32 \mathrm{~mL}, 0$. $321 \mathrm{mmol}, 1.0 \mathrm{M}$ in THF). The solution stirred at $-78{ }^{\circ} \mathrm{C}$ for $15 \mathrm{~min}$ before the dropwise addition of the aldehyde $18(78 \mathrm{mg}, 0.212 \mathrm{mmol})$ in THF $(2.0 \mathrm{~mL})$. The solution stirred for $45 \mathrm{~min}$ at $-78{ }^{\circ} \mathrm{C}$ at which point it was allowed to warm to room temperature and quenched with saturated aq $\mathrm{NH}_{4} \mathrm{Cl}(5 \mathrm{~mL})$. The mixture was extracted with ether $(2 \times 5$ $\mathrm{mL})$ and EtOAc $(2 \times 5 \mathrm{~mL})$ and the combined organic extracts were dried $\left(\mathrm{MgSO}_{4}\right)$, filtered and concentrated in vacuo and purified by flash column chromatography (silica gel, gradient elution 5-30\% EtOAc in hexanes) to yield $62 \mathrm{mg}(80 \%)$ of alkyne 19 as a colorless oil: ${ }^{1} \mathrm{H} \mathrm{NMR}\left(600 \mathrm{MHz}, \mathrm{CDCl}_{3}\right) \delta 4.38-$ $4.35(\mathrm{~m}, 2 \mathrm{H}) ; 2.78-2.72(\mathrm{~m}, 1 \mathrm{H}) ; 2.51-2.46(\mathrm{~m}, 1 \mathrm{H}) ; 2.37-2.33(\mathrm{~m}, 1 \mathrm{H}) ; 2.19-1.72(\mathrm{~m}, 12 \mathrm{H}), 0.89(\mathrm{~s}, 9 \mathrm{H})$, 0.07 (s, 3H), 0.05 (s, 3H); ${ }^{13} \mathrm{C}$ NMR (150 MHz, $\left.\mathrm{CDCl}_{3}\right) \delta 176.8,108.3,84.5,75.0,73.0,68.9,44.0,43.1$, 40.1 , 35.0, 29.1, 28.5, 26.0, 19.2, 18.2, 17.2, -4.3, -5.1; HRMS (ESI) calc'd for $\mathrm{C}_{20} \mathrm{H}_{33} \mathrm{O}_{4} \mathrm{Si}[\mathrm{M}+\mathrm{H}]+$ : 365.2143; found: 365.2121.

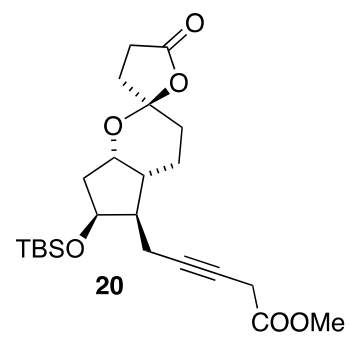

Alkynoate 20: To a solution of the alkyne 19 (49 $\mathrm{mg}, 0.135 \mathrm{mmol})$ in $\mathrm{MeCN}$ (1.5 $\mathrm{mL}$ ) was added Cul (3 $\mathrm{mg}, 0.013 \mathrm{mmol}$ ) followed by a solution of methyl diazoacetate (162 mg, $0.202 \mathrm{mmol}, 12.5 \%$ by weight in $\mathrm{CH}_{2} \mathrm{Cl}_{2}$ ). The solution stirred overnight and concentrated in vacuo. Purification by flash chromatography (silica gel, gradient elution 2-20\% EtOAc in hexanes) to yield $44 \mathrm{mg}(75 \%)$ of methyl ester 20 as a colorless oil: ${ }^{1} \mathrm{H}$ NMR $\left(400 \mathrm{MHz}, \mathrm{CDCl}_{3}\right) \delta 4.38-4.32(\mathrm{~m}$, 
2H), $3.73(\mathrm{~s}, 3 \mathrm{H}), 3.24(\mathrm{app} \mathrm{t}, J=2.5 \mathrm{~Hz}, 2 \mathrm{H}), 2.80-2.69(\mathrm{~m}, 1 \mathrm{H}), 2.47(\mathrm{dd}, J=9.6,2.7 \mathrm{~Hz}, 1 \mathrm{H}), 2.35(\mathrm{~m}$, $1 \mathrm{H}), 2.23-1.79(\mathrm{~m}, 10 \mathrm{H}), 1.79-1.67(\mathrm{~m}, 1 \mathrm{H}), 0.86(\mathrm{~s}, 9 \mathrm{H}), 0.05(\mathrm{~s}, 3 \mathrm{H}), 0.03(\mathrm{~s}, 3 \mathrm{H}) ;{ }^{13} \mathrm{C} \mathrm{NMR}(100 \mathrm{MHz}$, $\left.\mathrm{CDCl}_{3}\right) \delta 176.7,169.4,108.4,83.6,75.1,73.1,71.2,52.6,44.0,43.1,40.1,35.0,30.5,29.1,26.0,25.9$, 19.2, 18.1, 17.5, -4.4, -5.0; HRMS (ESI) calc'd for $\mathrm{C}_{23} \mathrm{H}_{37} \mathrm{O}_{6} \mathrm{Si}[\mathrm{M}+\mathrm{H}]+:$ 437.2354; found: 437.2348.

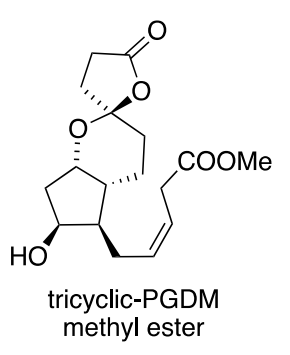

tricyclic-PGDM methyl ester: To a solution of alkynoate $20(9 \mathrm{mg}, 0.021 \mathrm{mmol})$ in $\operatorname{MeCN}(1.0 \mathrm{~mL})$ at $0{ }^{\circ} \mathrm{C}$ was added $70 \%$ HF-pyridine $(13 \mu \mathrm{L}, 0.52 \mathrm{mmol}, 2.5$ equiv). The reaction was allowed to slowly warm to room temperature. After $2 \mathrm{~h}$, additional HF-pyridine ( $13 \mu \mathrm{L}, 0.52 \mathrm{mmol}, 2.5$ equiv) was added. The solution stirred for $2 \mathrm{~h}$, at which point it was quenched with saturated aq $\mathrm{NaHCO}_{3}(8 \mathrm{~mL})$. The product was extracted from the aqueous layer with EtOAc $(3 \times 3 \mathrm{~mL})$ and $\mathrm{CHCl}_{3}(3 \times 3 \mathrm{~mL})$, dried $\left(\mathrm{MgSO}_{4}\right)$, filtered, and concentrated in vacuo. To a separate reaction flask was added Lindlar's catalyst (5 $\mathrm{mg}$ ) and $\mathrm{MeOH}(0.5 \mathrm{~mL})$ at room temperature. The vessel was purged with hydrogen gas $(3 \mathrm{x})$ before the crude product was added in $\mathrm{MeOH}(0.5 \mathrm{~mL})$. The reaction stirred under one atmosphere of hydrogen overnight at which point it was filtered through Celite and concentrated in vacuo. The resultant residue was purified by flash chromatography (silica gel, gradient elution 2- 20\% EtOAc in hexanes) to yield $1 \mathrm{mg}(15 \%)$ of tricyclic-PGDM methyl ester as a colorless oil: ${ }^{1} \mathrm{H}$ NMR $\left(600 \mathrm{MHz}, \mathrm{CDCl}_{3}\right) \delta 5.68($ app t, $\mathrm{J}=5.68 \mathrm{~Hz}$, 1H), 5.57 (app q, $J=6.8 \mathrm{~Hz}, 1 \mathrm{H}), 4.41(\operatorname{app~t}, J=4.7 \mathrm{~Hz}, 1 \mathrm{H}), 4.34-4.29(\mathrm{~m}, 1 \mathrm{H}), 3.70(\mathrm{~s}, 3 \mathrm{H}), 3.29(\mathrm{dd}, J=$ 14.7, 8.6 Hz, 1H), 3.03 (dd, $J=16.0,6.2 \mathrm{~Hz}, 1 \mathrm{H}), 2.76(\mathrm{dt}, J=17.9,9.7 \mathrm{~Hz}, 1 \mathrm{H}), 2.52-2.43(\mathrm{~m}, 2 \mathrm{H}), 2.37-$ $1.61(\mathrm{~m}, 11 \mathrm{H}) ;{ }^{13} \mathrm{C}$ NMR $\left(150 \mathrm{MHz}, \mathrm{CDCl}_{3}\right) \delta 176.7,173.2,133.1,121.8,108.2,75.1,72.0,52.4,43.8$, 42.3, 39.5, 35.0, 33.1, 28.9, 28.5, 25.2,18.5; HRMS (ESI) calc'd for $\mathrm{C}_{17} \mathrm{H}_{25} \mathrm{O}_{6}[\mathrm{M}+\mathrm{H}]+$ : 325.1646; found: 325.1644 . 


\section{Copy of ${ }^{1} \mathrm{H}$ and ${ }^{13} \mathrm{C}$ NMR Spectra}

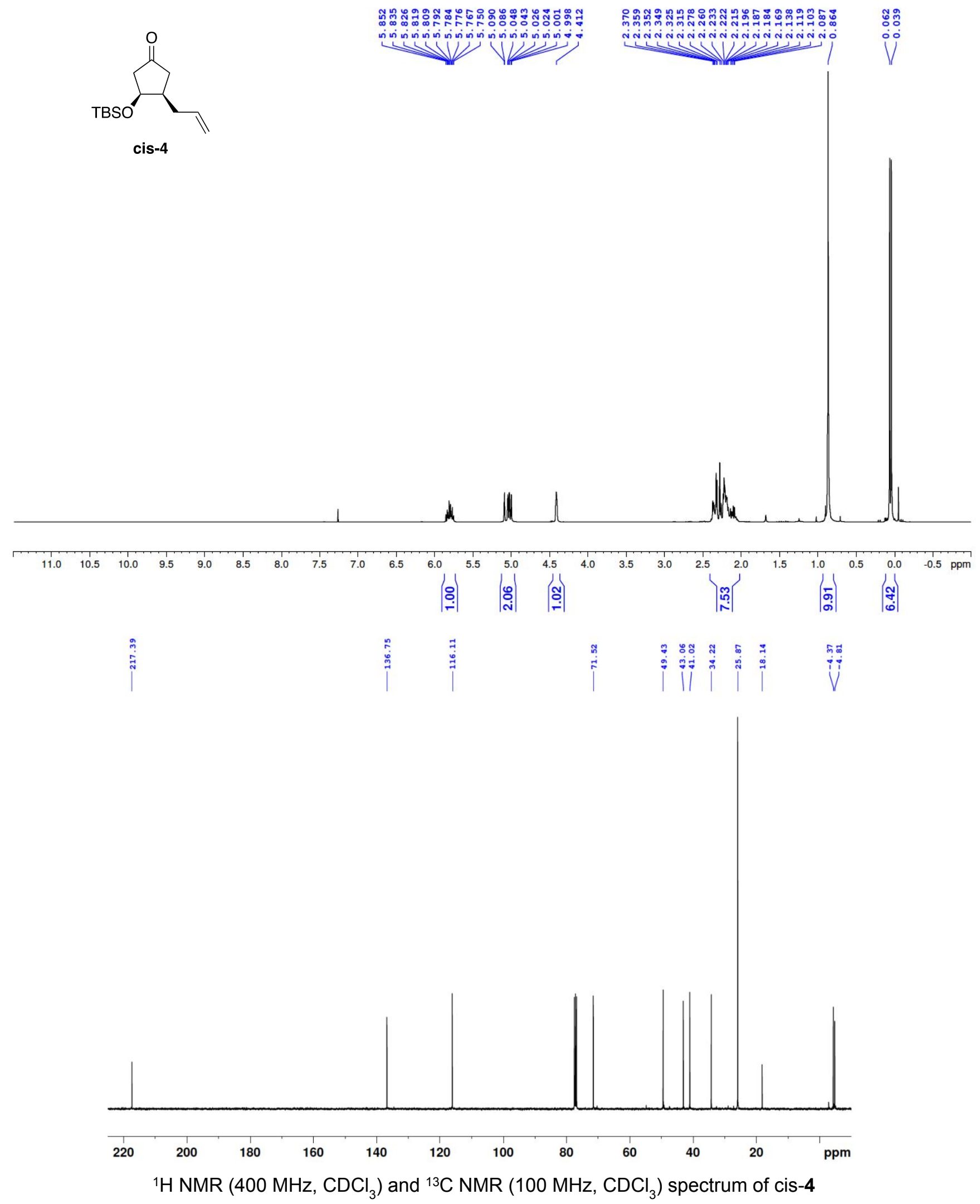




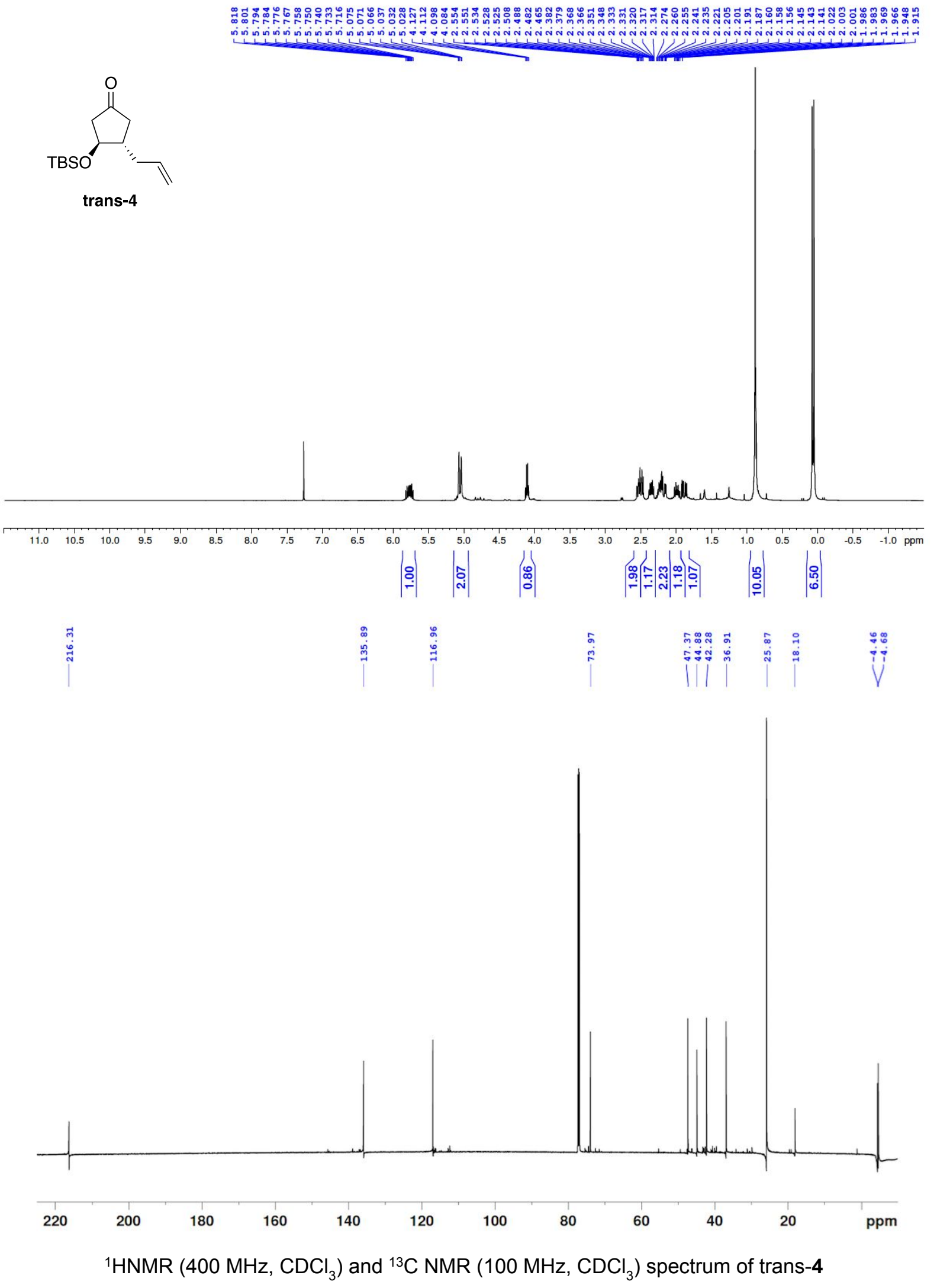




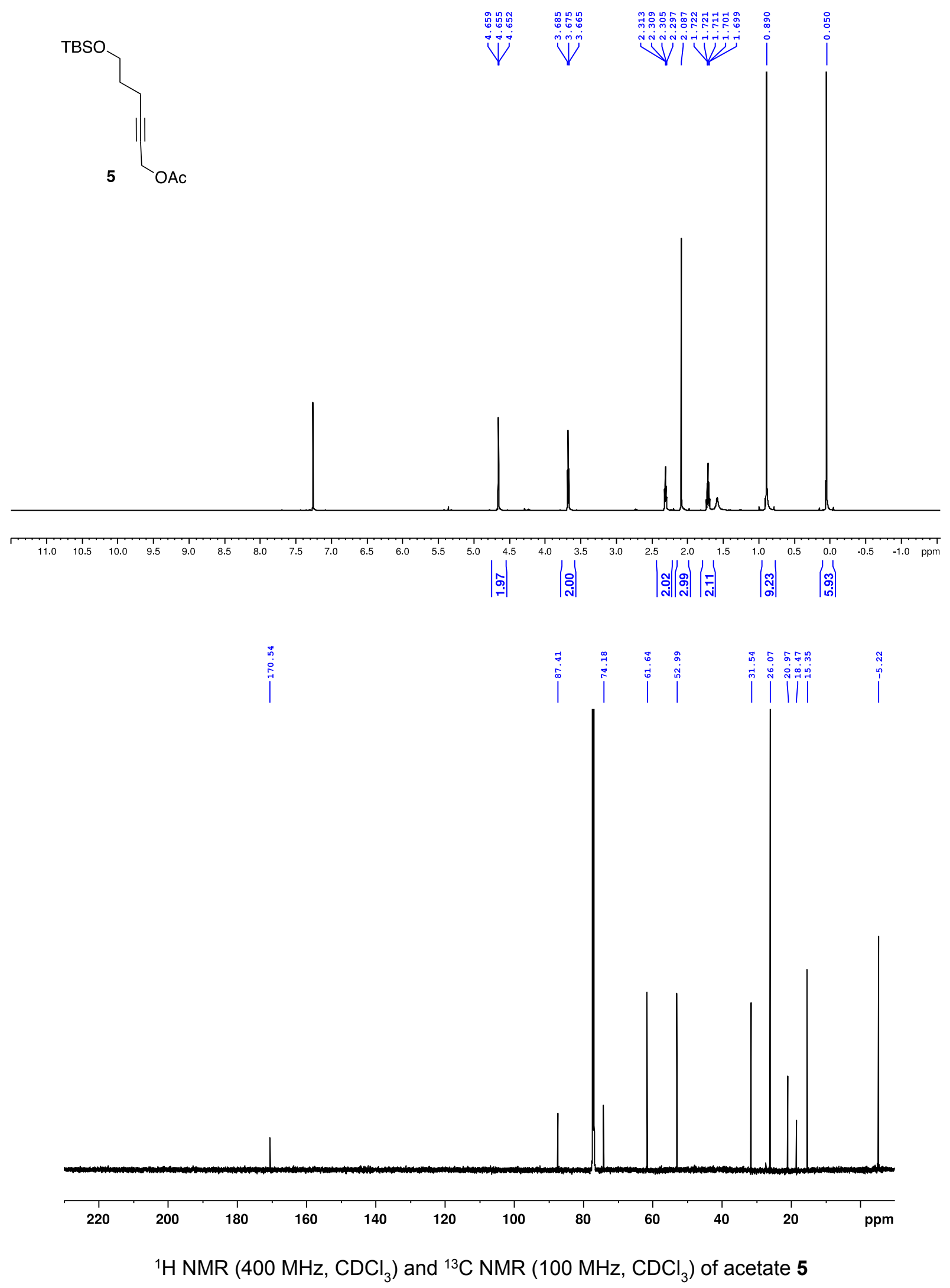



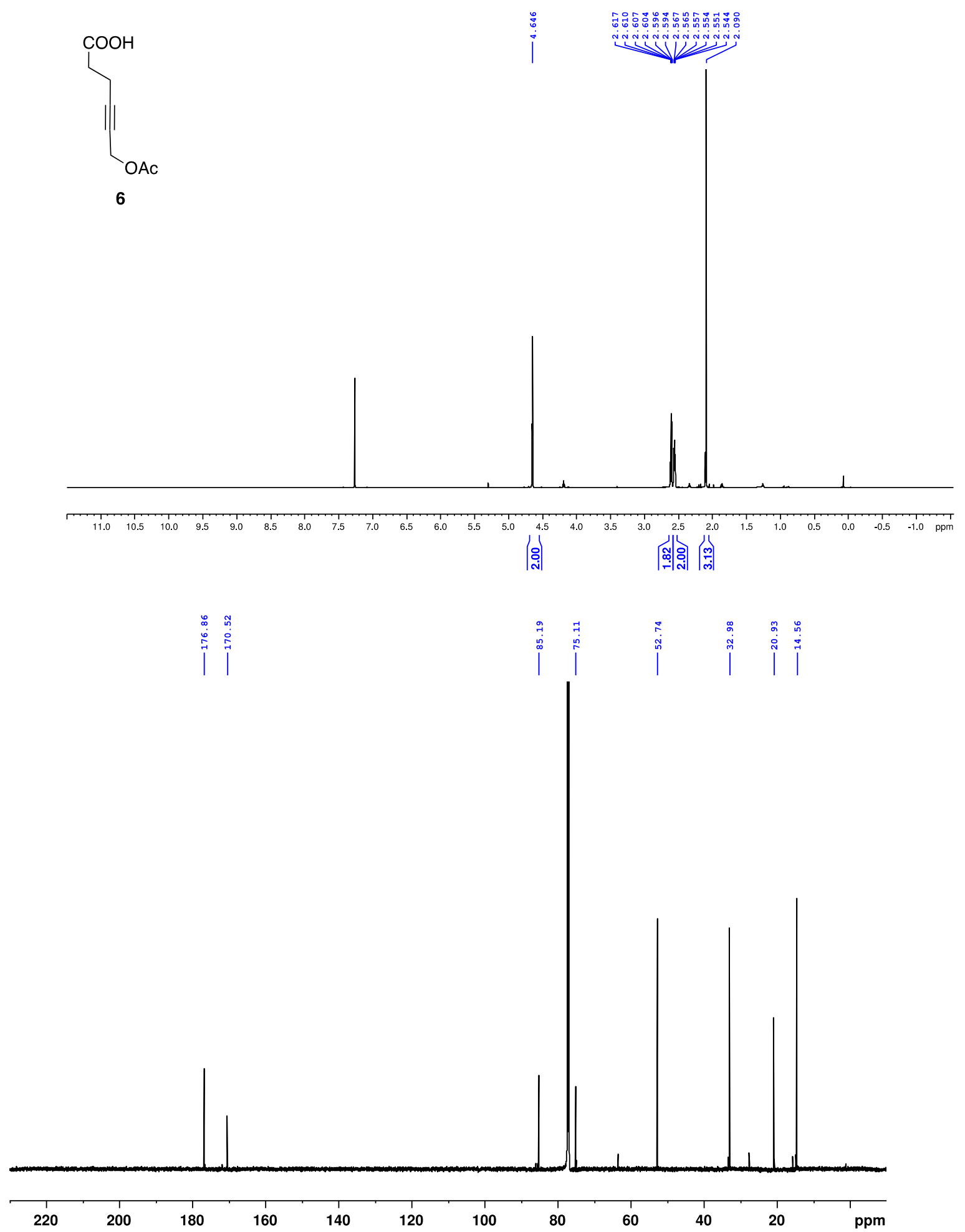

${ }^{1} \mathrm{H} \mathrm{NMR}\left(400 \mathrm{MHz}, \mathrm{CDCl}_{3}\right)$ and ${ }^{13} \mathrm{C} \mathrm{NMR}\left(100 \mathrm{MHz}, \mathrm{CDCl}_{3}\right)$ spectrum of acid 6 


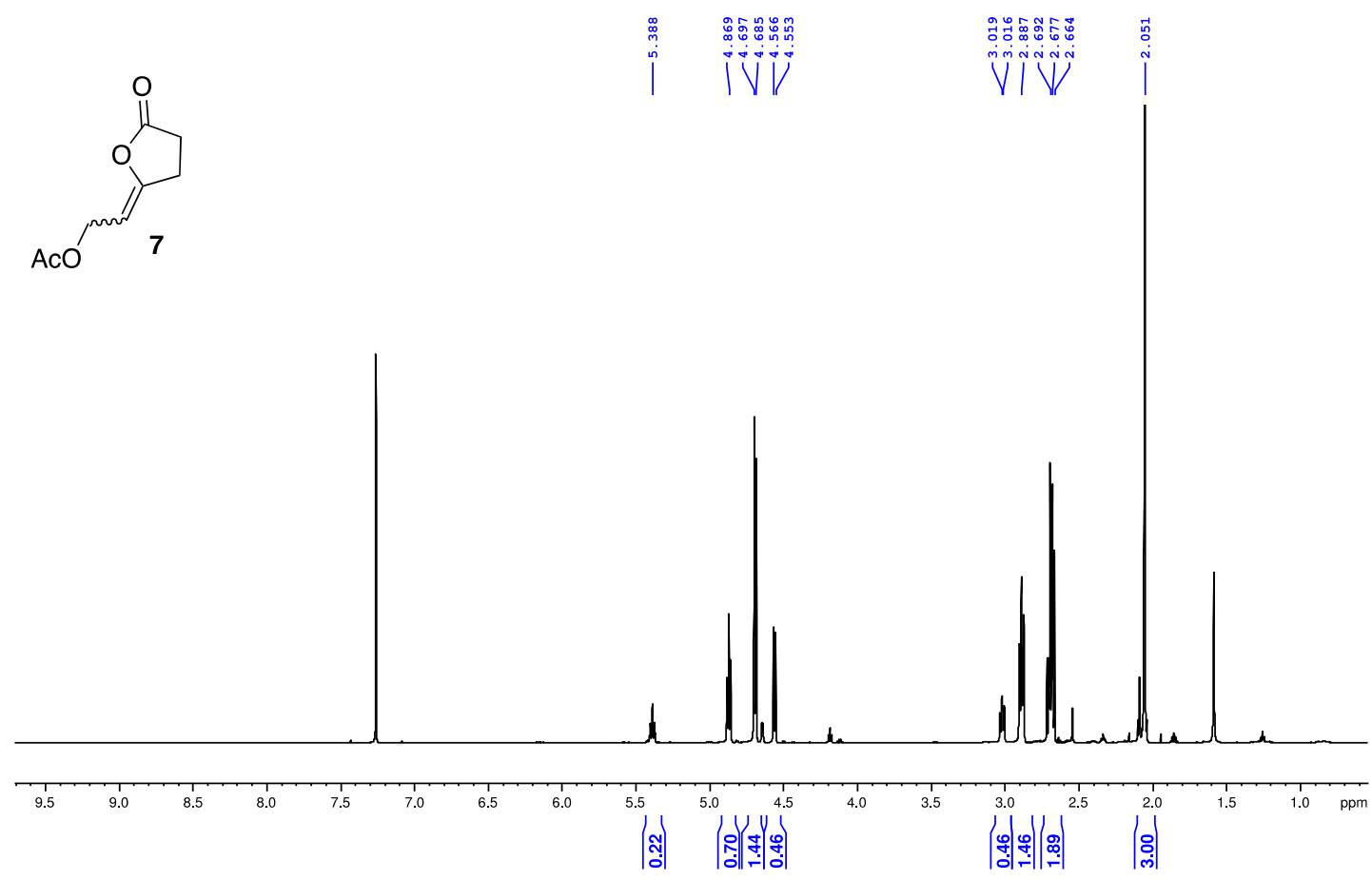

${ }^{1} \mathrm{H}$ NMR $\left(400 \mathrm{MHz}, \mathrm{CDCl}_{3}\right)$ spectrum of acetate 7 

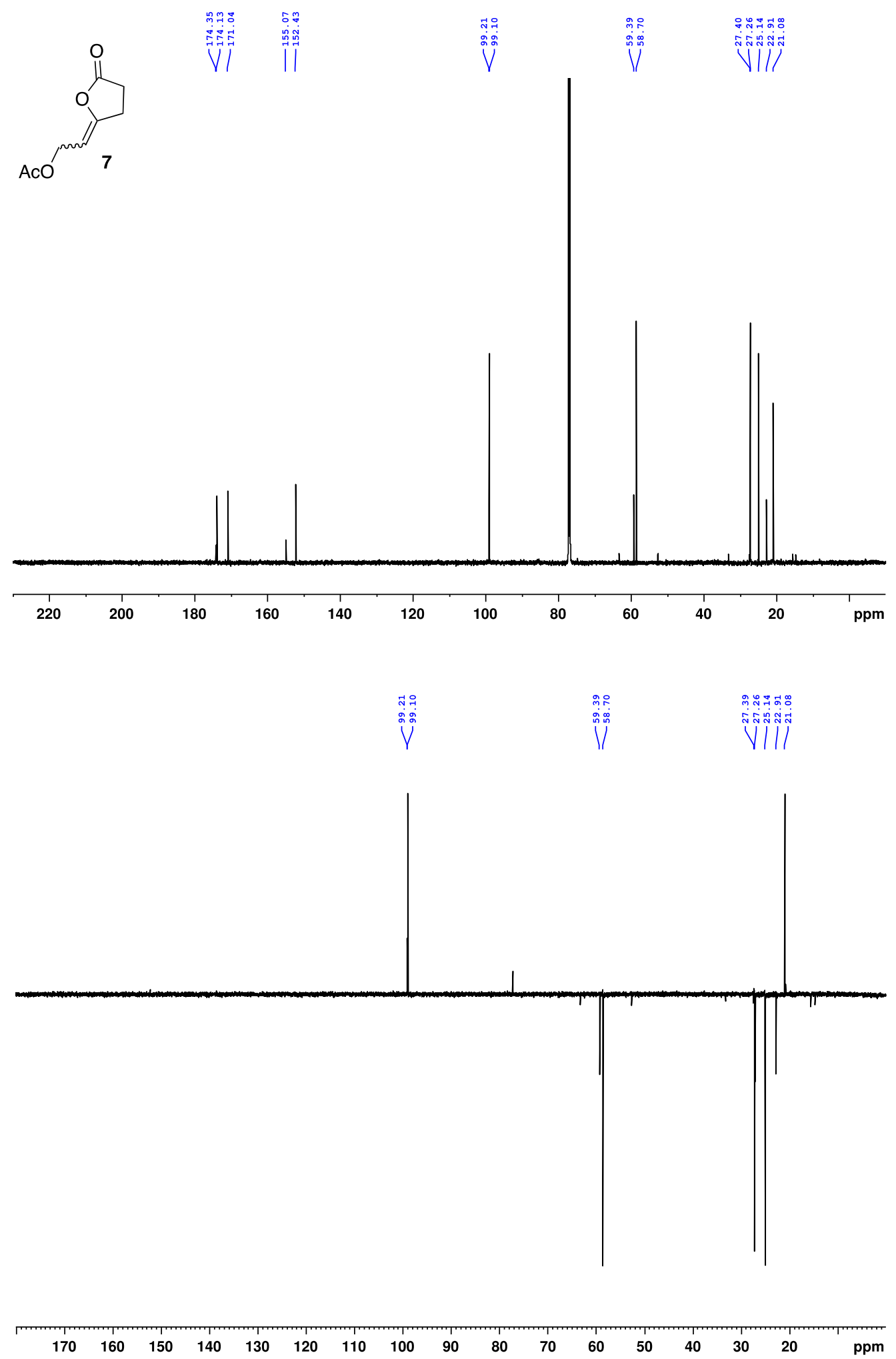

${ }^{13} \mathrm{C} \mathrm{NMR}\left(100 \mathrm{MHz}, \mathrm{CDCl}_{3}\right)$ and DEPT NMR $\left(100 \mathrm{MHz}, \mathrm{CDCl}_{3}\right)$ of spectrum acetate 7 


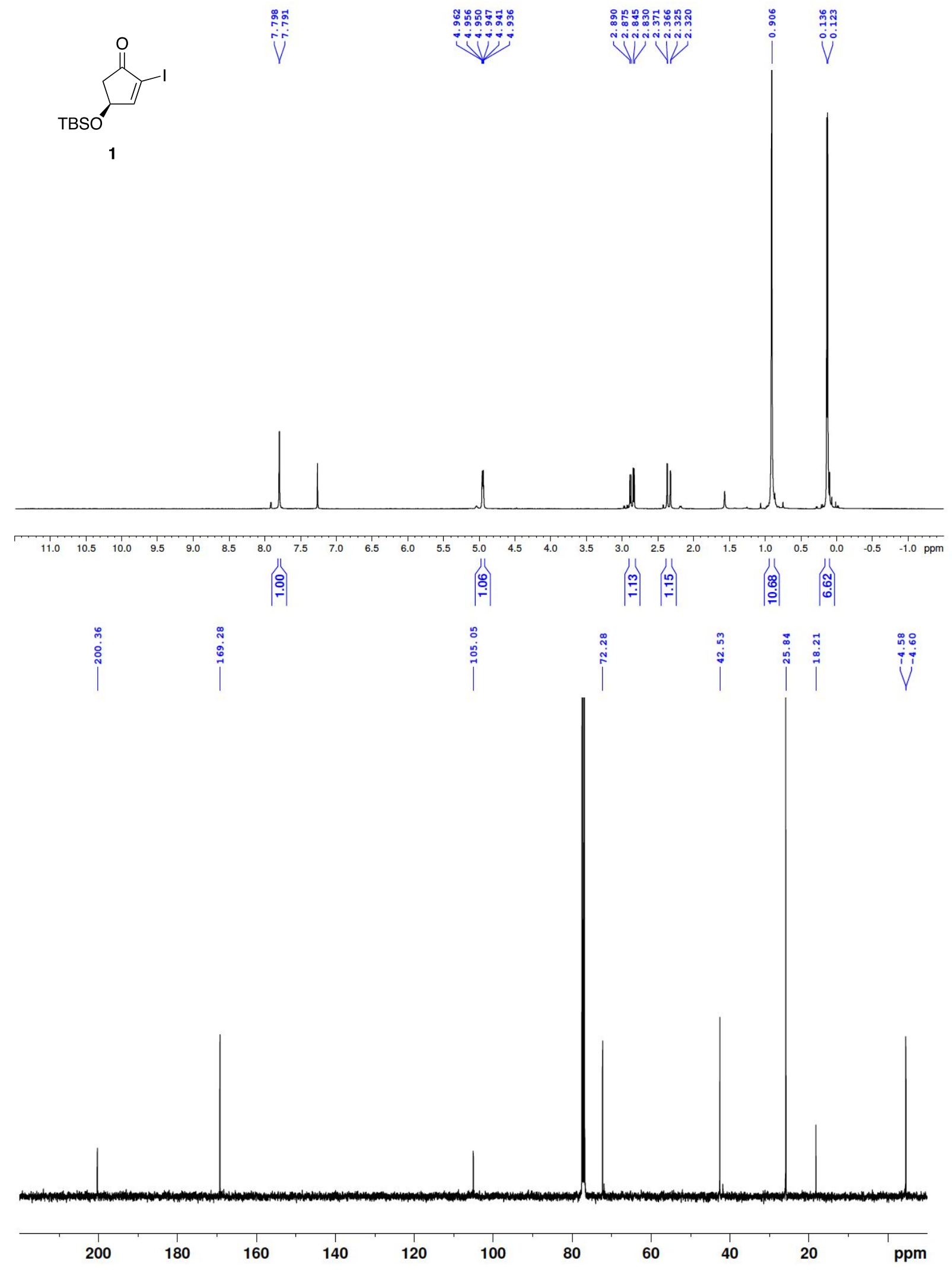

${ }^{1} \mathrm{H} \mathrm{NMR}\left(400 \mathrm{MHz}, \mathrm{CDCl}_{3}\right)$ and ${ }^{13} \mathrm{C} \mathrm{NMR}\left(100 \mathrm{MHz}, \mathrm{CDCl}_{3}\right)$ spectrum of iodoenone 1 


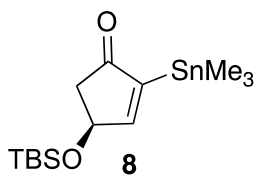

Vั
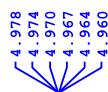

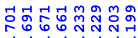

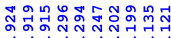
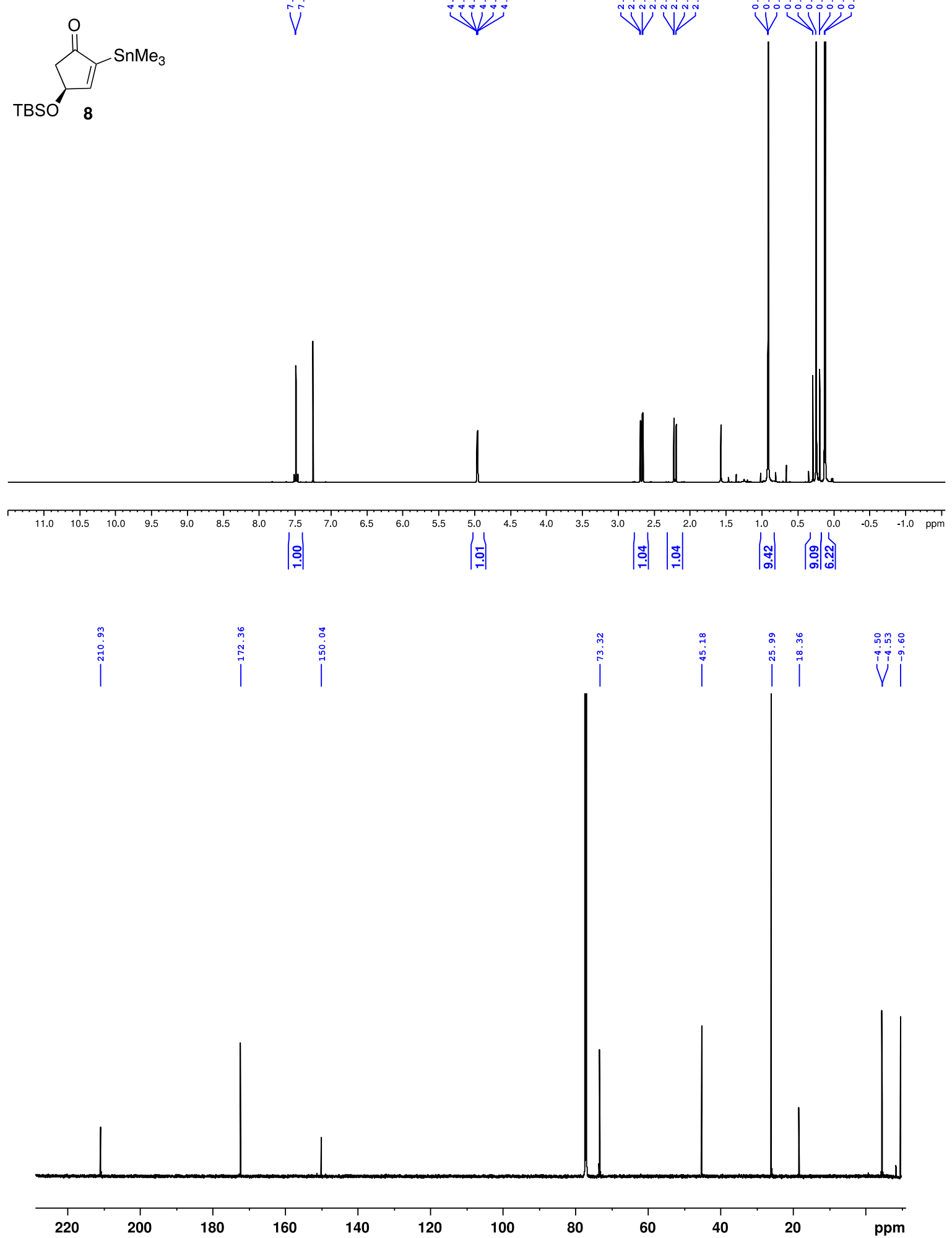

${ }^{1} \mathrm{H}$ NMR $\left(600 \mathrm{MHz}, \mathrm{CDCl}_{3}\right)$ and ${ }^{13} \mathrm{C} \mathrm{NMR}\left(150 \mathrm{MHz}, \mathrm{CDCl}_{3}\right)$ spectrum of stannenone 8 

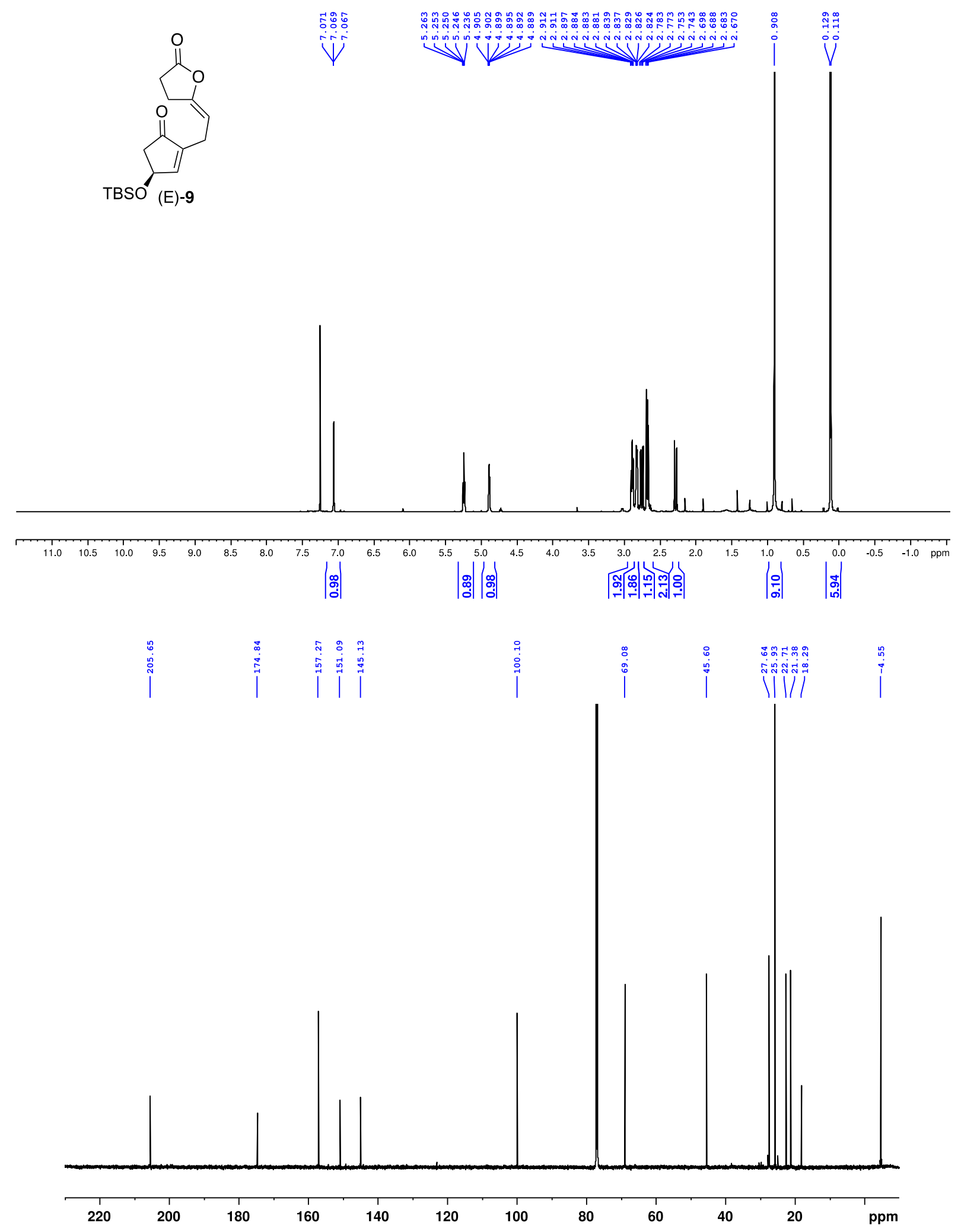

${ }^{1} \mathrm{H}$ NMR $\left(600 \mathrm{MHz}, \mathrm{CDCl}_{3}\right)$ and ${ }^{13} \mathrm{C}$ NMR $\left(150 \mathrm{MHz}, \mathrm{CDCl}_{3}\right)$ spectrum of (E)-9 


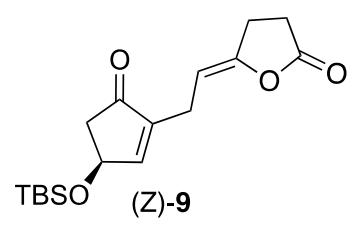

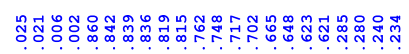

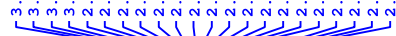

皮

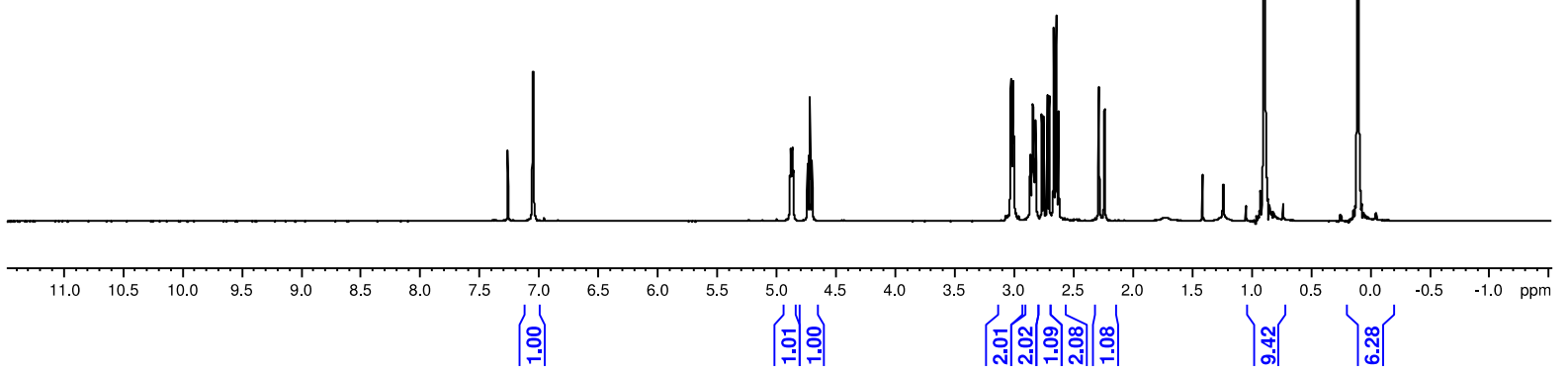

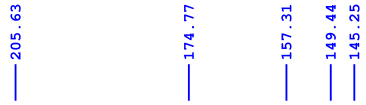

$\mid$
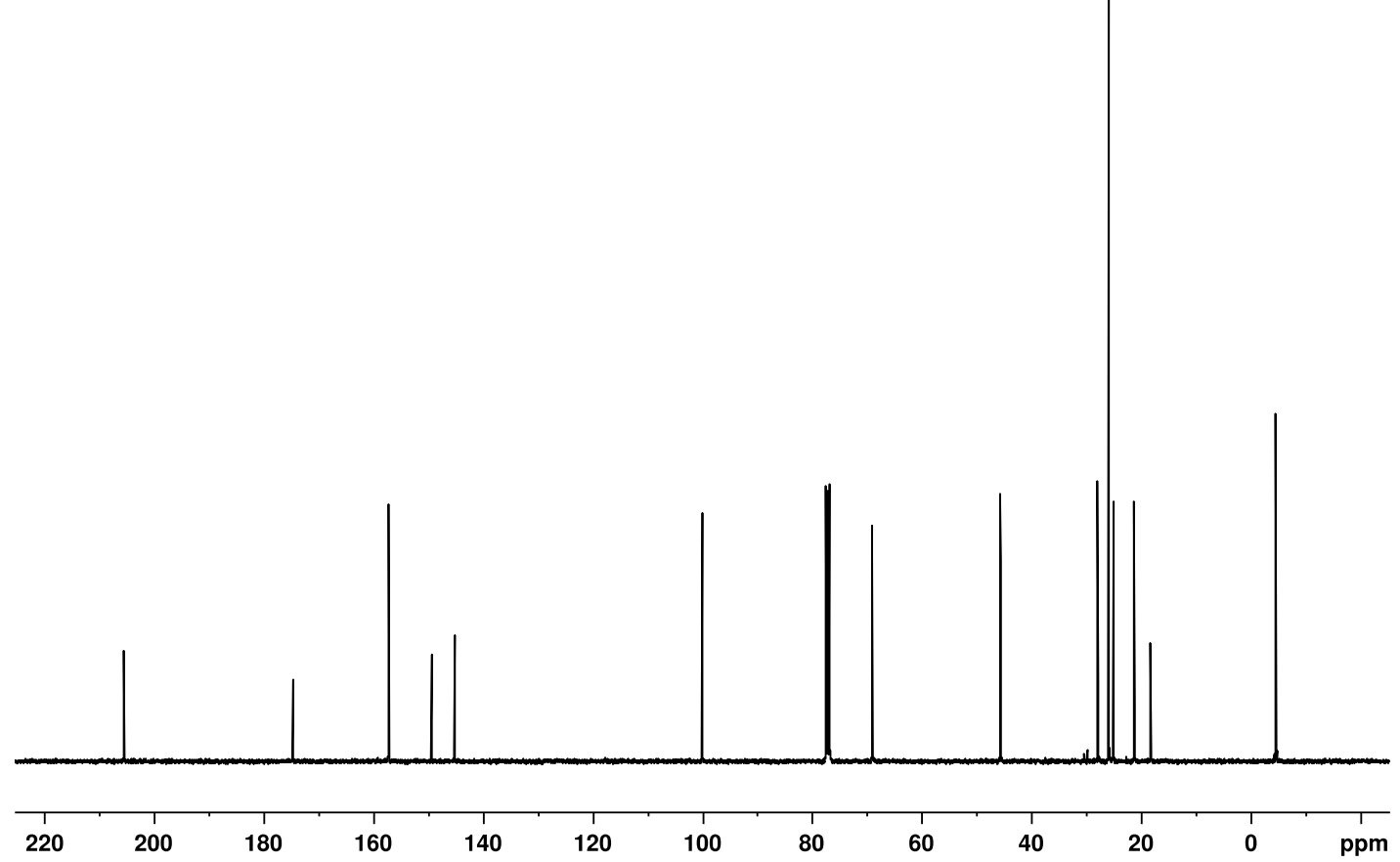

${ }^{1} \mathrm{H} \mathrm{NMR}\left(600 \mathrm{MHz}, \mathrm{CDCl}_{3}\right)$ and ${ }^{13} \mathrm{C}$ NMR $\left(150 \mathrm{MHz}, \mathrm{CDCl}_{3}\right)$ spectrum of $(\mathrm{Z})-9$ 

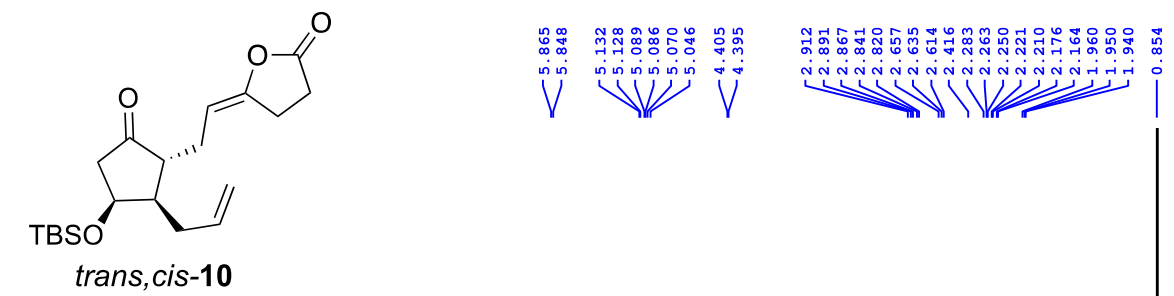

trans, cis-10
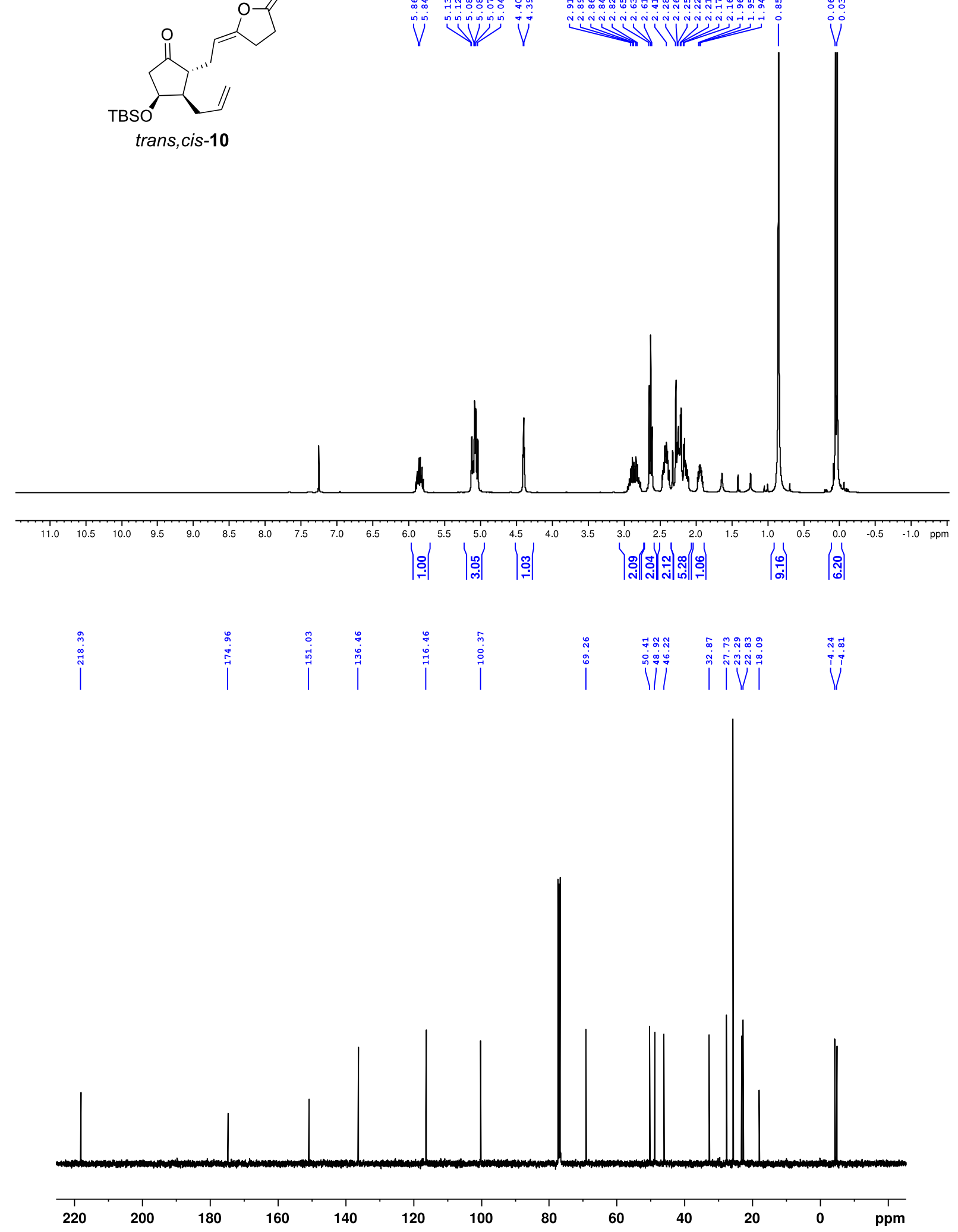

${ }^{1} \mathrm{H} \mathrm{NMR}\left(600 \mathrm{MHz}, \mathrm{CDCl}_{3}\right)$ and ${ }^{13} \mathrm{C}$ NMR $\left(150 \mathrm{MHz}, \mathrm{CDCl}_{3}\right)$ spectrum of trans, cis-10 


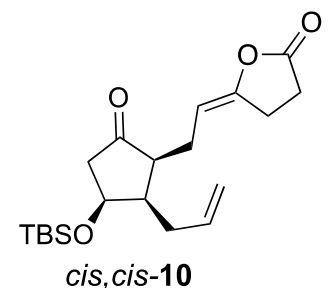

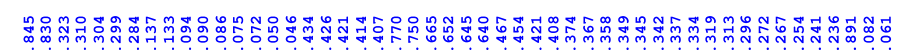

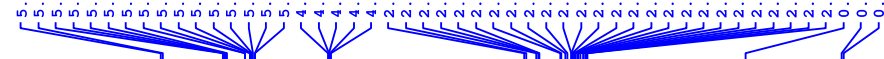

cis, cis-10

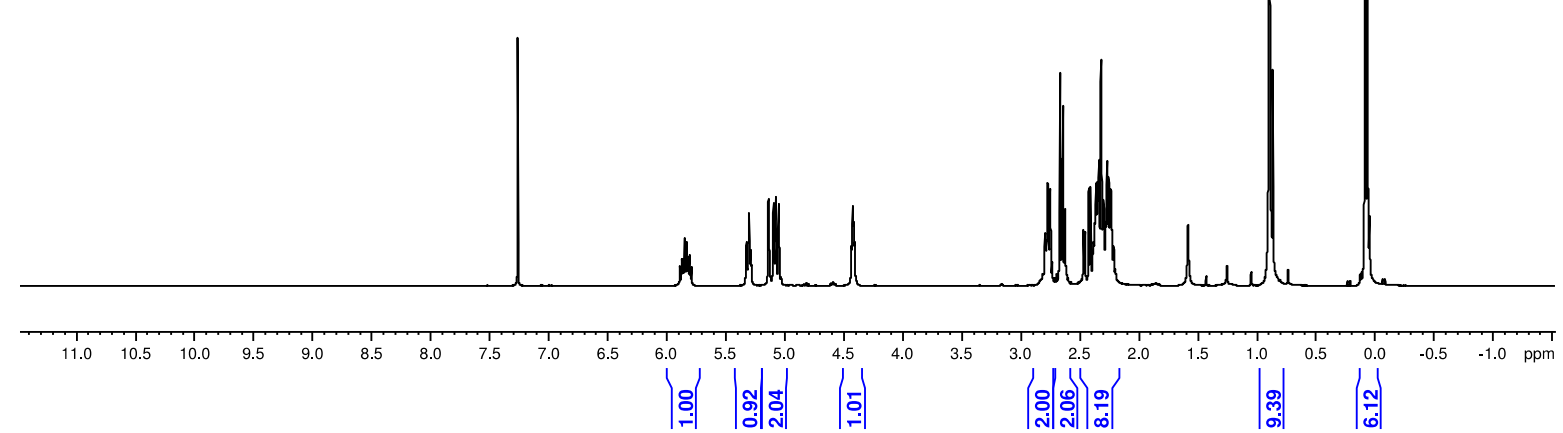

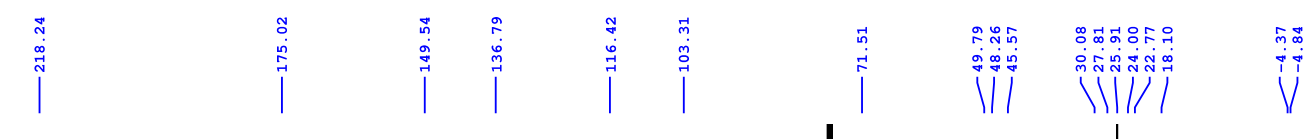

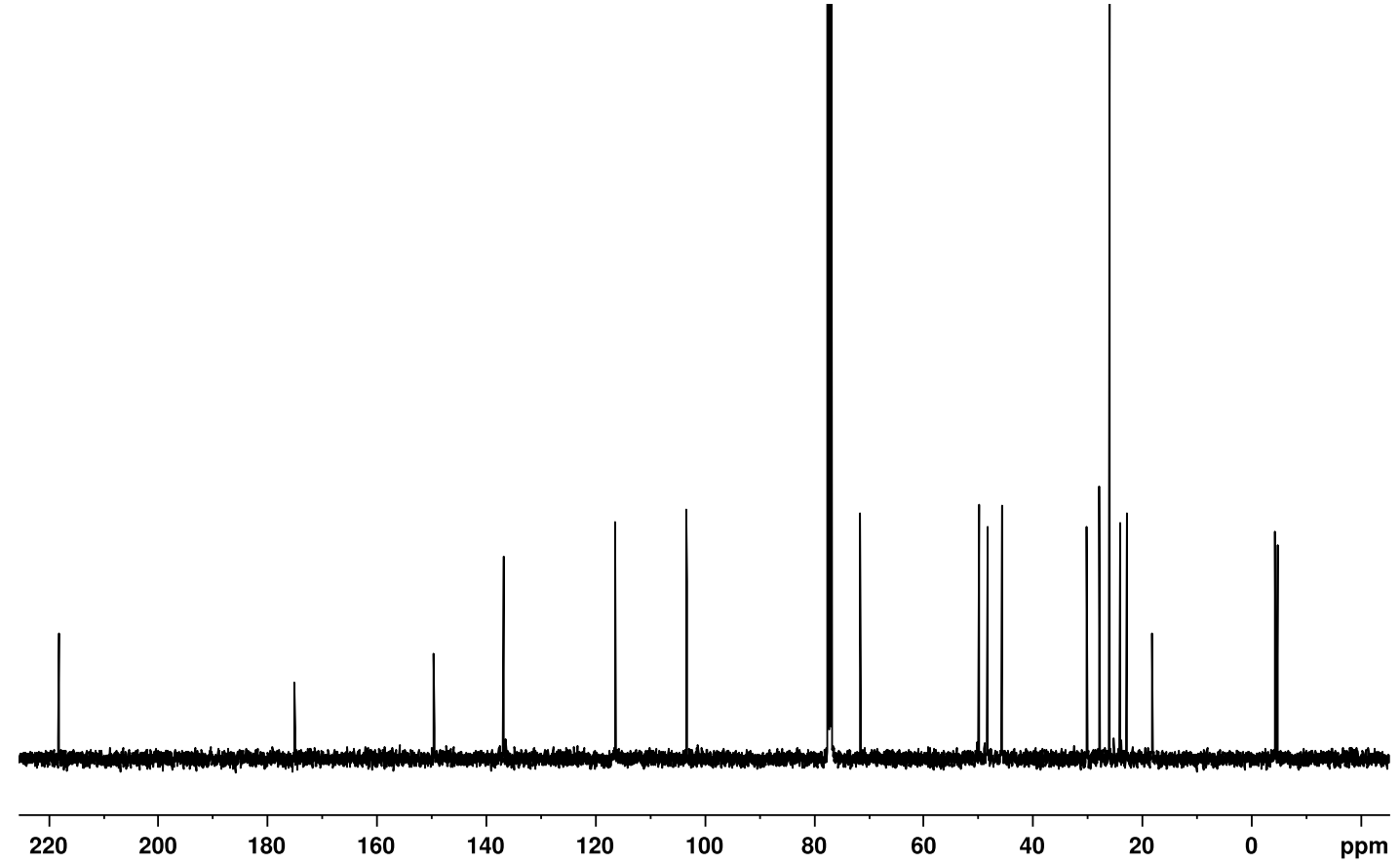

${ }^{1} \mathrm{H} \mathrm{NMR}\left(600 \mathrm{MHz}, \mathrm{CDCl}_{3}\right)$ and ${ }^{13} \mathrm{C} \mathrm{NMR}\left(150 \mathrm{MHz}, \mathrm{CDCl}_{3}\right)$ spectrum of cis, cis-10 

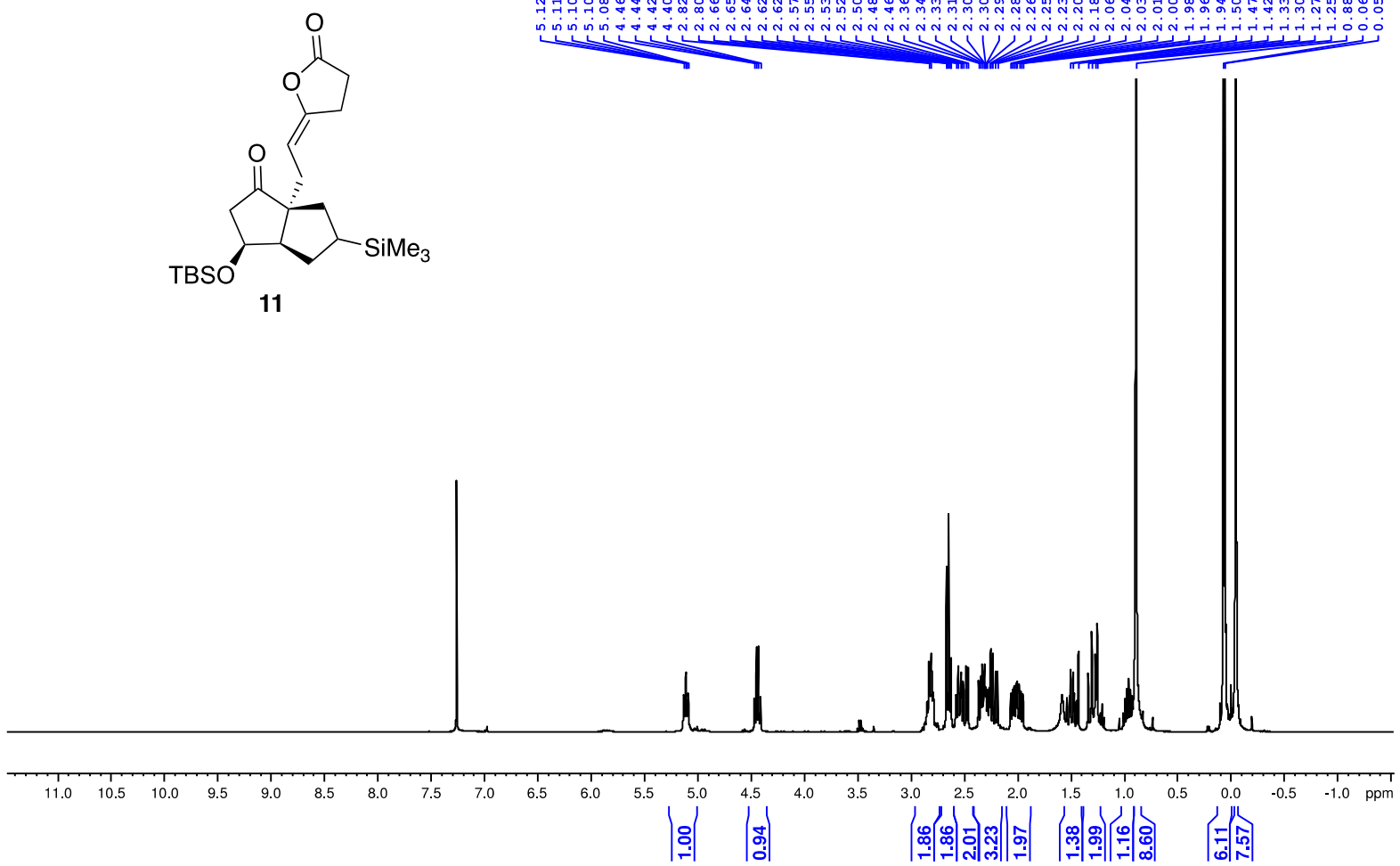

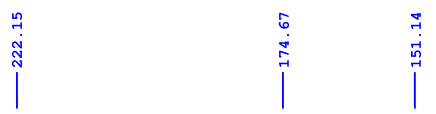

$\stackrel{\substack{+\vdots}}{\stackrel{1}{+}}$

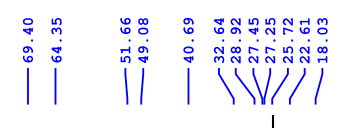

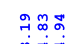

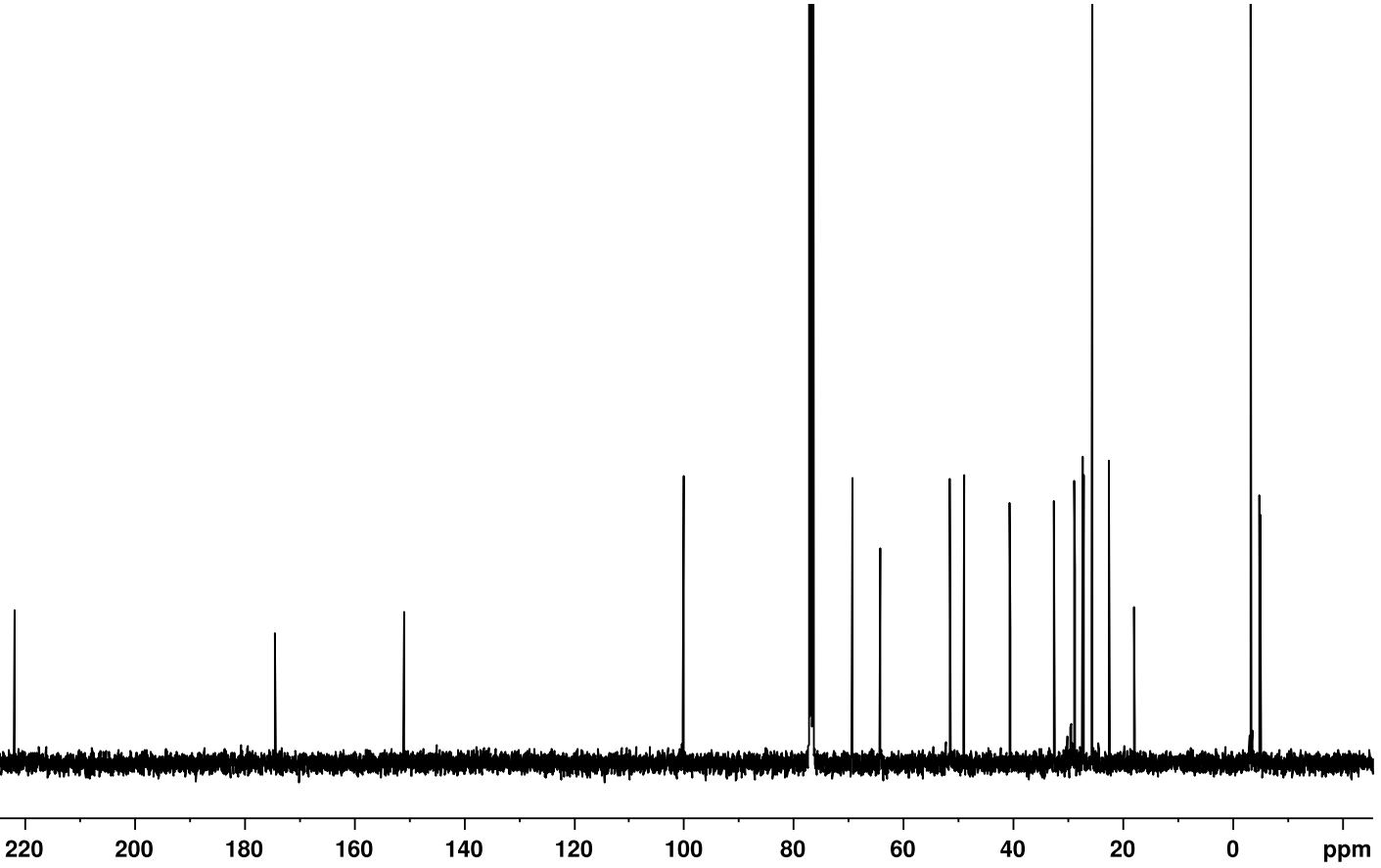

${ }^{1} \mathrm{H}$ NMR $\left(600 \mathrm{MHz}, \mathrm{CDCl}_{3}\right)$ and ${ }^{13} \mathrm{C} \mathrm{NMR}\left(150 \mathrm{MHz}, \mathrm{CDCl}_{3}\right)$ spectrum of cyclopentanone 11 


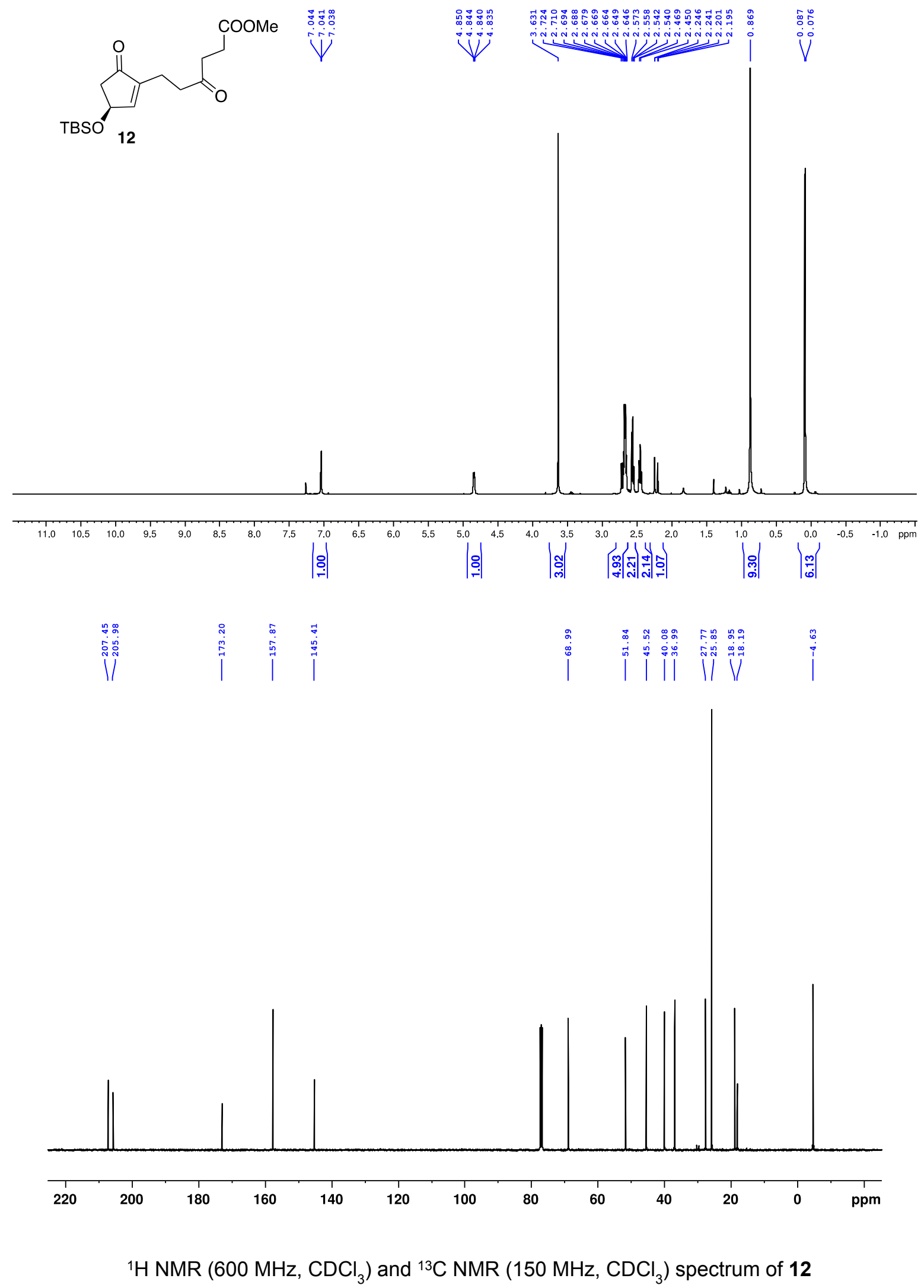




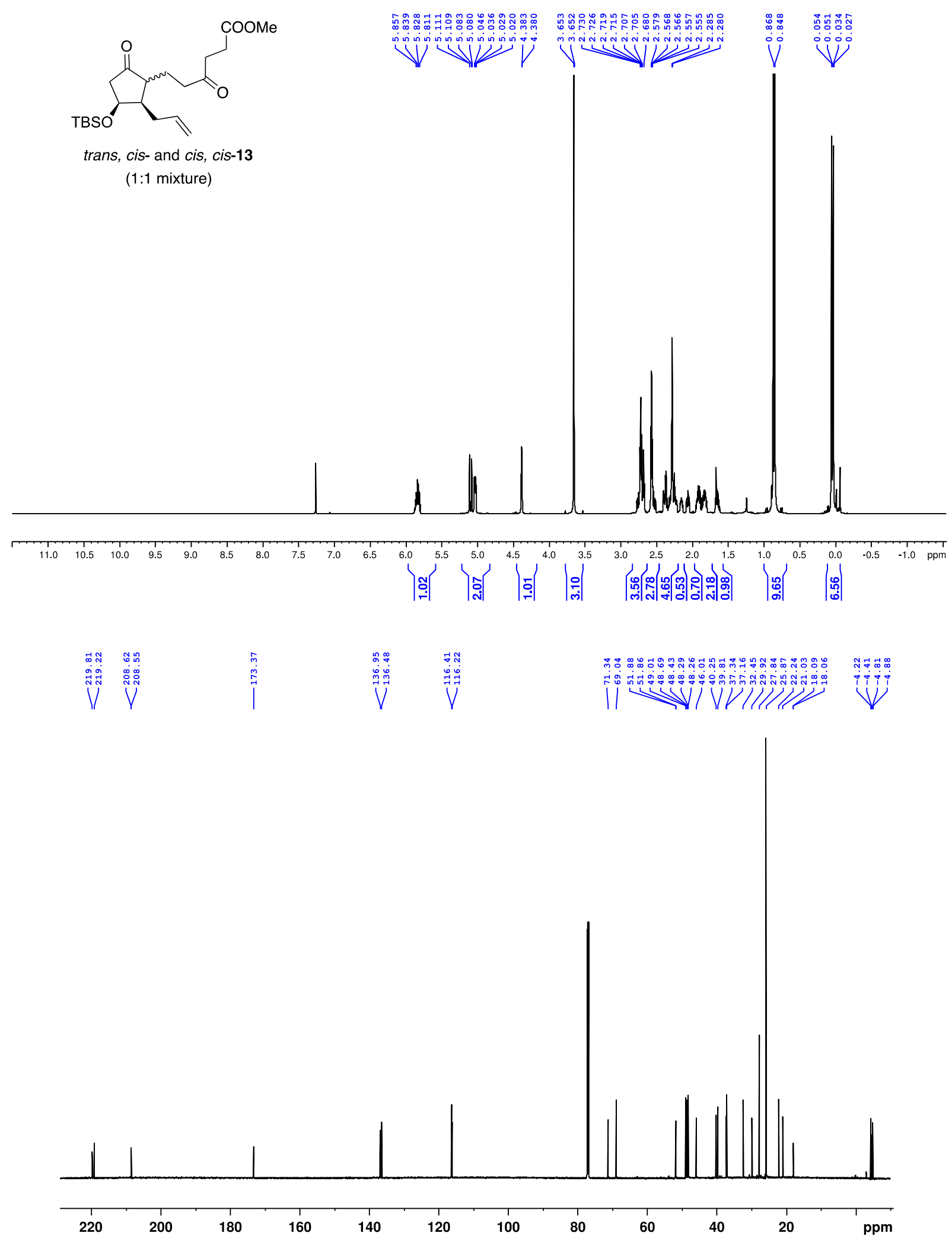

${ }^{1} \mathrm{H} \mathrm{NMR}\left(600 \mathrm{MHz}, \mathrm{CDCl}_{3}\right)$ and ${ }^{13} \mathrm{C} \mathrm{NMR}\left(150 \mathrm{MHz}, \mathrm{CDCl}_{3}\right)$ spectrum of trans, cis and cis, cis- 13 
Supporting Information<smiles></smiles>

trans, cis- 13

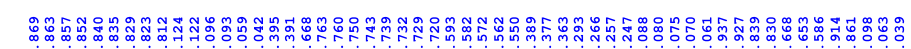

:
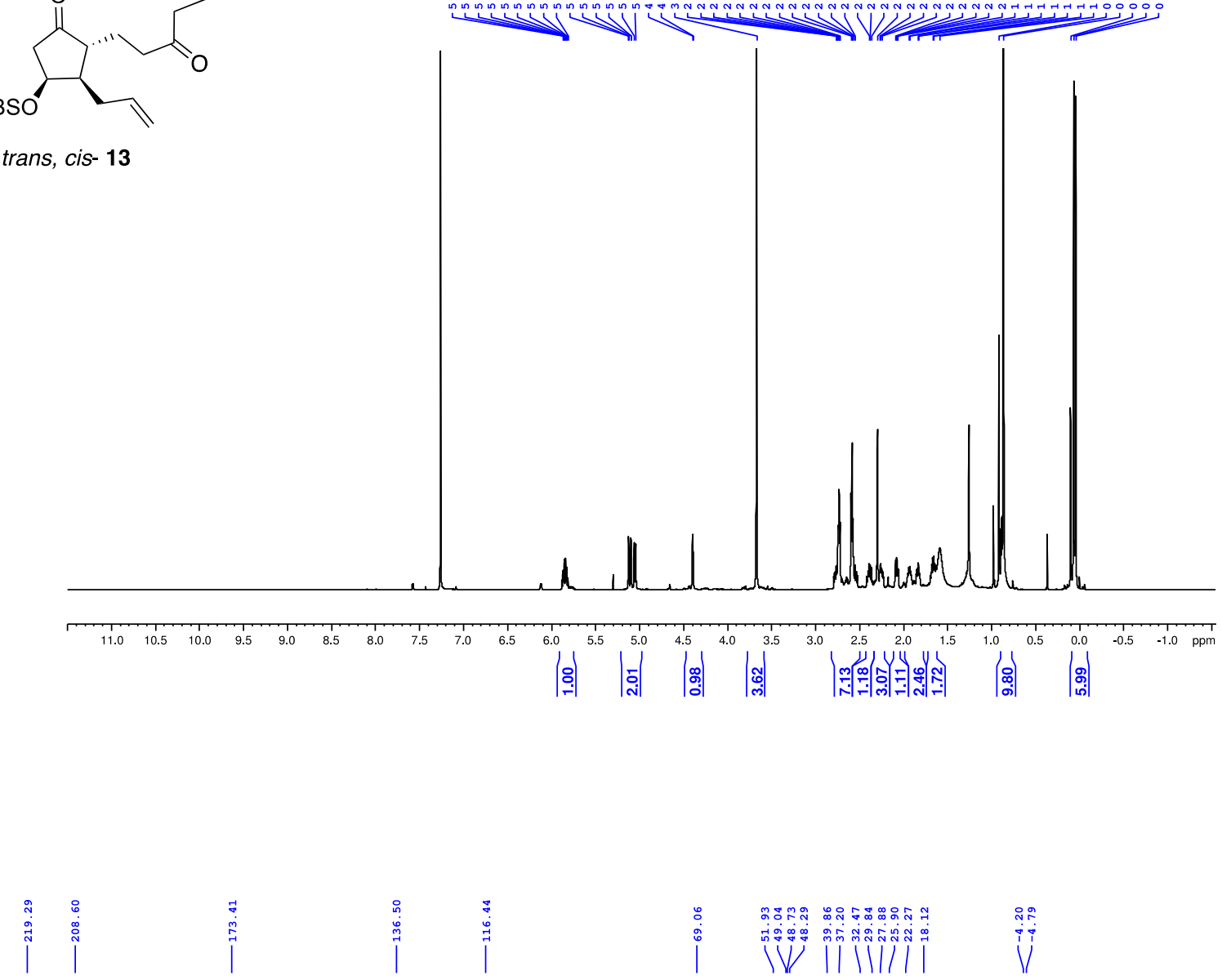

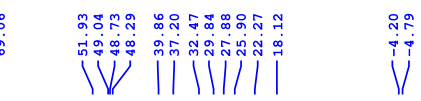

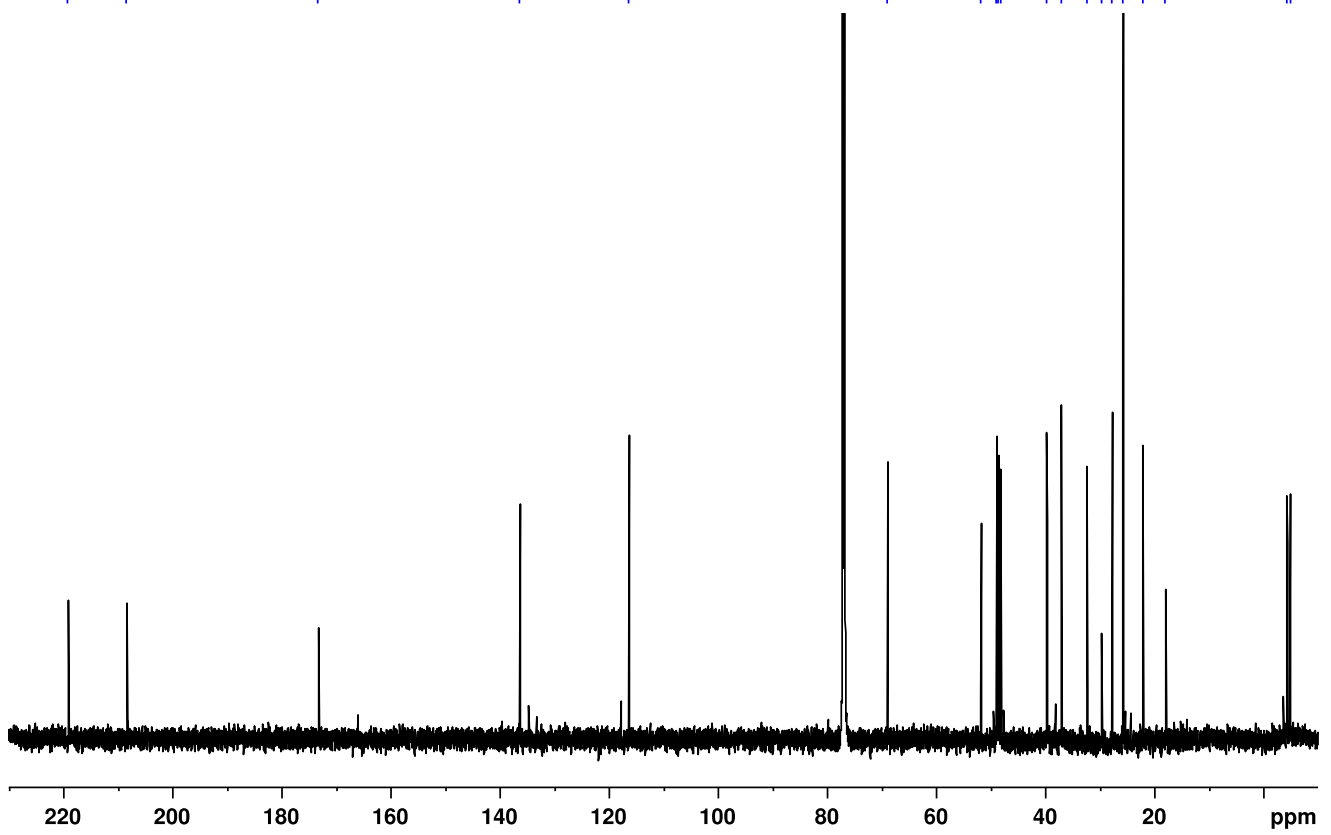

${ }^{1} \mathrm{H}$ NMR $\left(600 \mathrm{MHz}, \mathrm{CDCl}_{3}\right)$ and ${ }^{13} \mathrm{C} \mathrm{NMR}\left(150 \mathrm{MHz}, \mathrm{CDCl}_{3}\right)$ spectrum of trans, cis- 13 

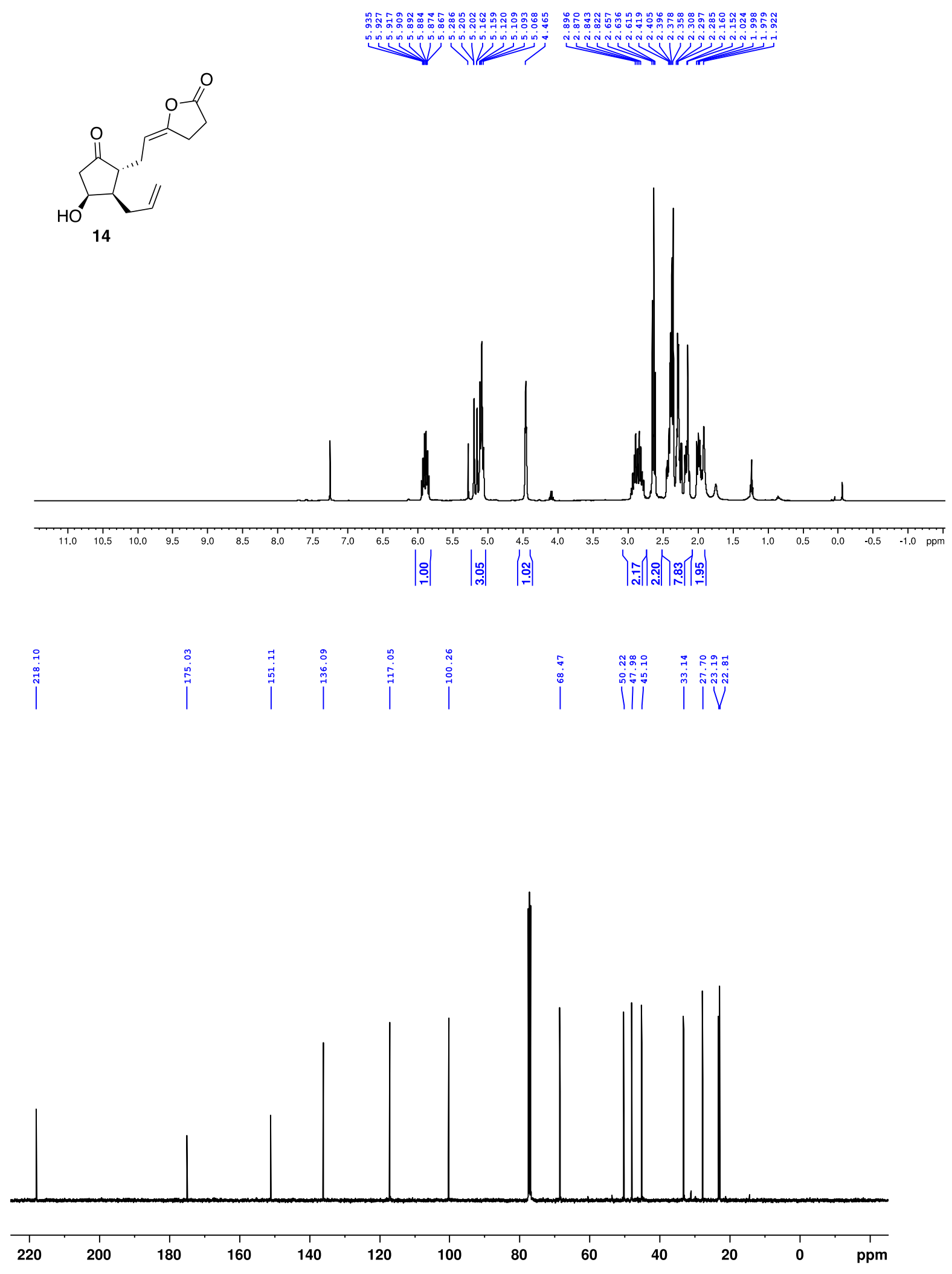

${ }^{1} \mathrm{H} \mathrm{NMR}\left(600 \mathrm{MHz}, \mathrm{CDCl}_{3}\right)$ and ${ }^{13} \mathrm{C} \mathrm{NMR}\left(150 \mathrm{MHz}, \mathrm{CDCl}_{3}\right)$ spectrum of 14 


\section{Supporting Information}
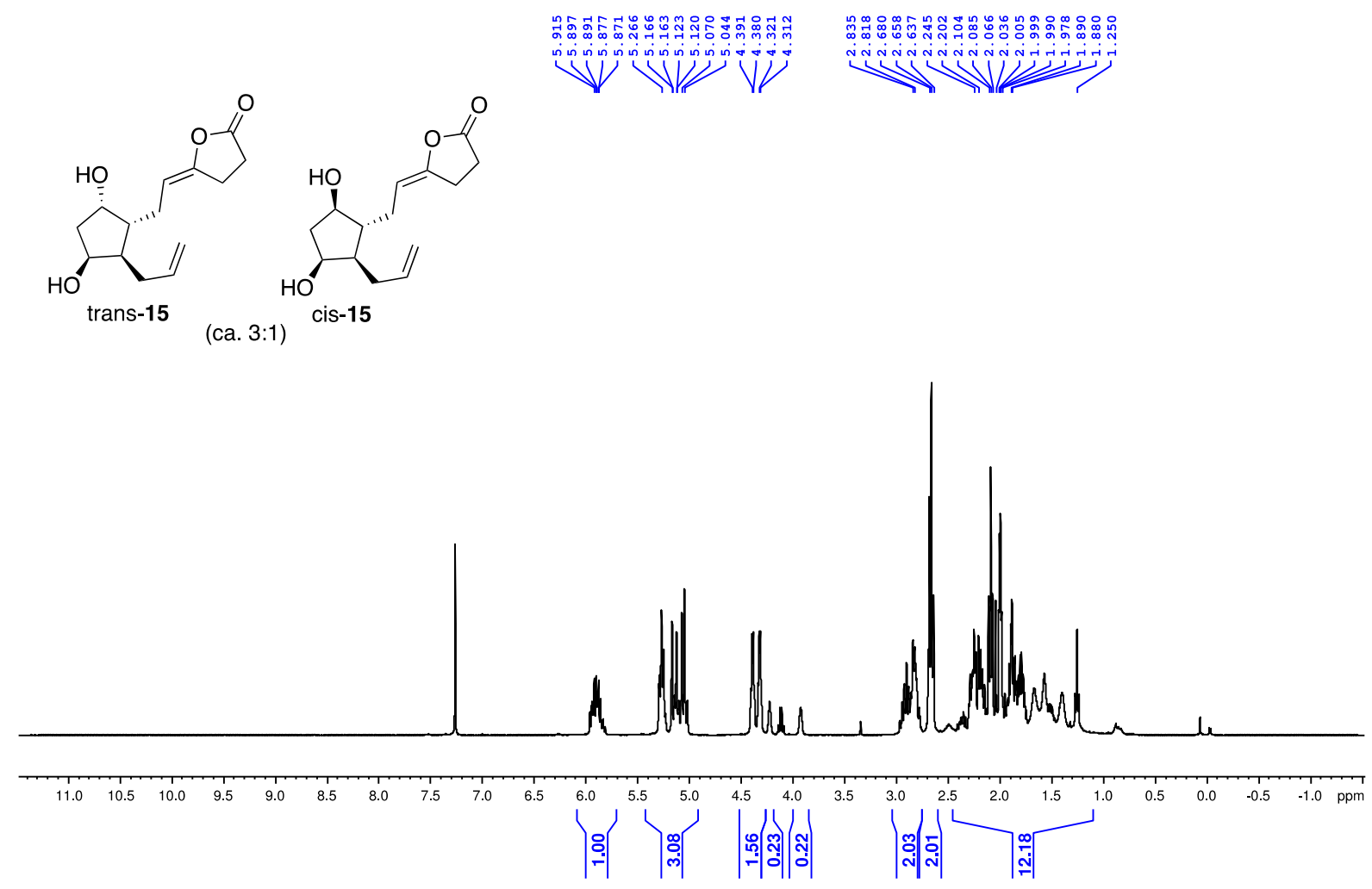

${ }^{1} \mathrm{H} \mathrm{NMR}\left(600 \mathrm{MHz}, \mathrm{CDCl}_{3}\right)$ spectrum of trans- and cis-15 (ca. 3:1) 

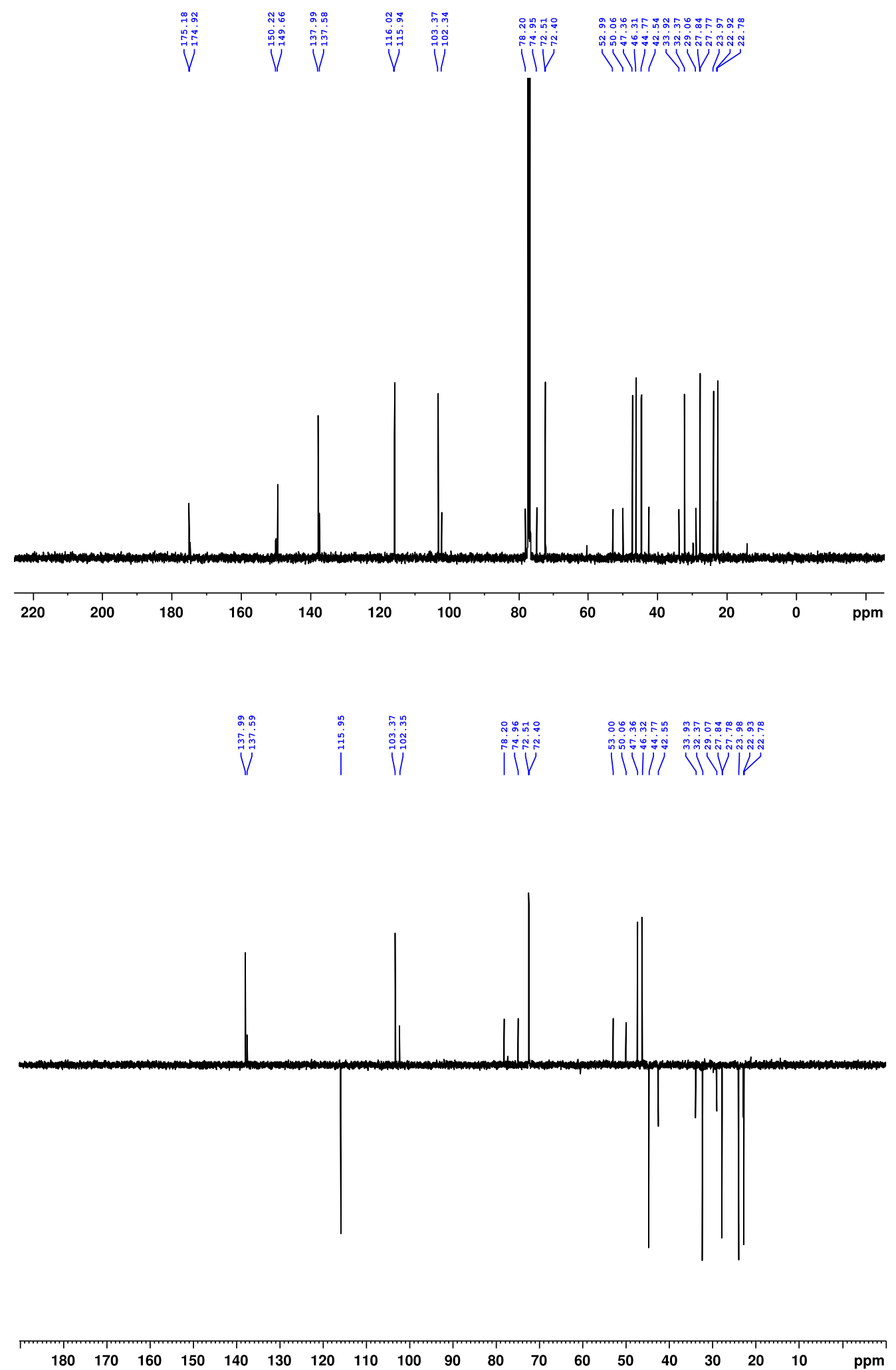

${ }^{13} \mathrm{C}$ NMR $\left(150 \mathrm{MHz}, \mathrm{CDCl}_{3}\right.$ ) and DEPT (150 MHz, $\mathrm{CDCl}_{3}$ ) NMR spectrum of trans- and cis-15 (ca. 3:1) 

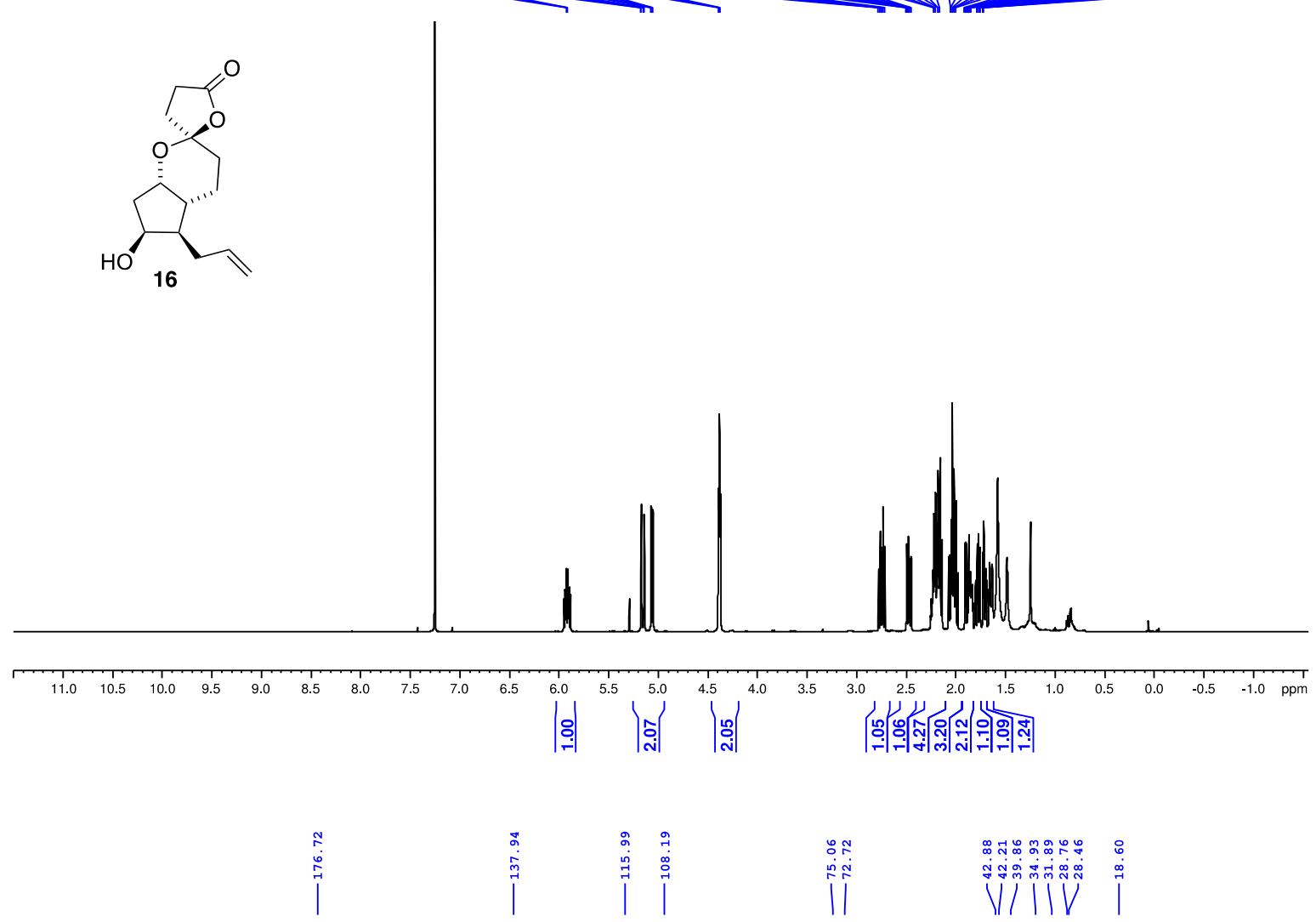

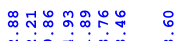

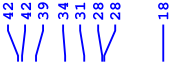

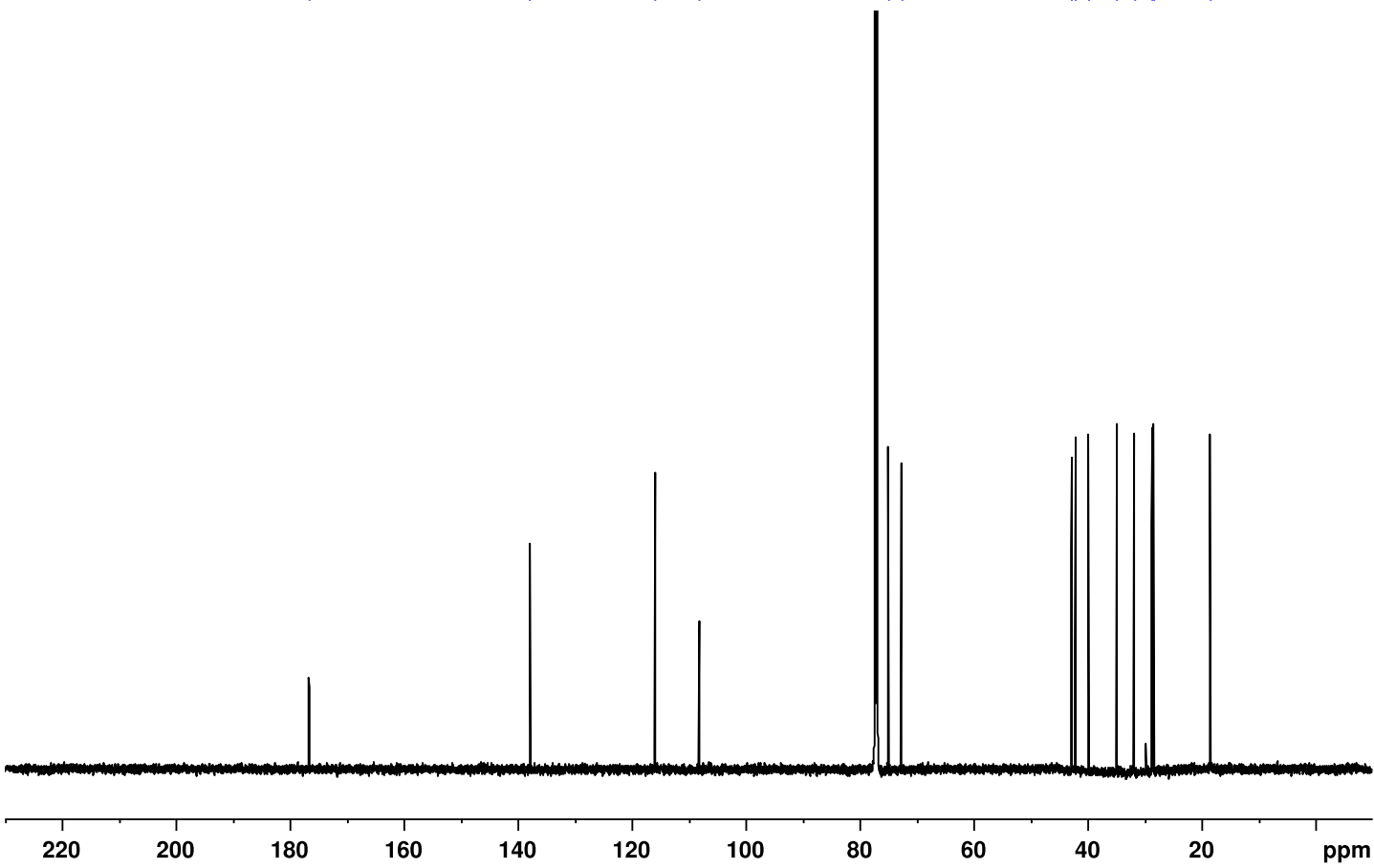

${ }^{1} \mathrm{H} \mathrm{NMR}\left(600 \mathrm{MHz}, \mathrm{CDCl}_{3}\right)$ and ${ }^{13} \mathrm{C} \mathrm{NMR}\left(150 \mathrm{MHz}, \mathrm{CDCl}_{3}\right)$ spectrum of 16 


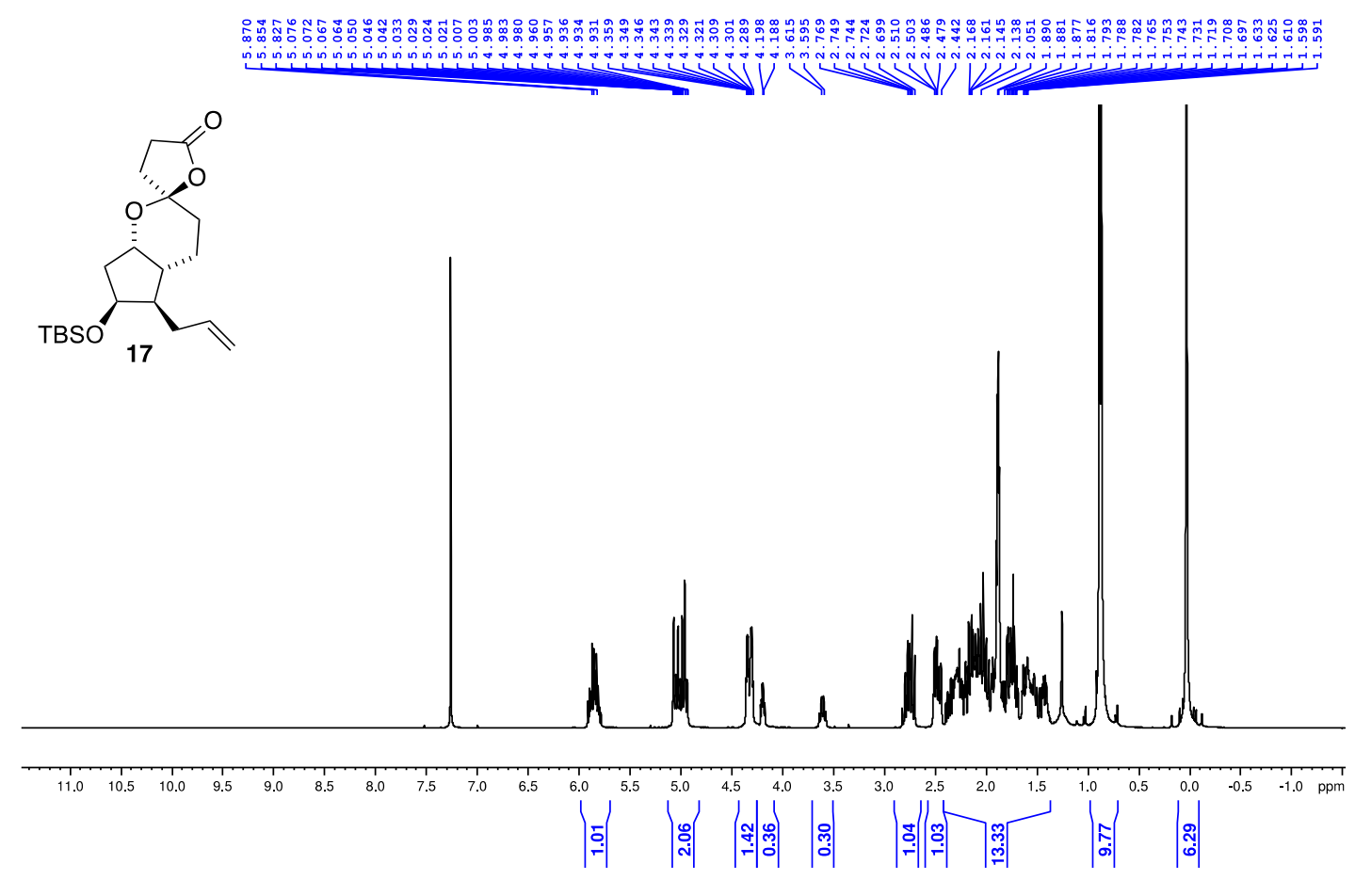

${ }^{1} \mathrm{H}$ NMR $\left(600 \mathrm{MHz}, \mathrm{CDCl}_{3}\right)$ spectrum of 17 

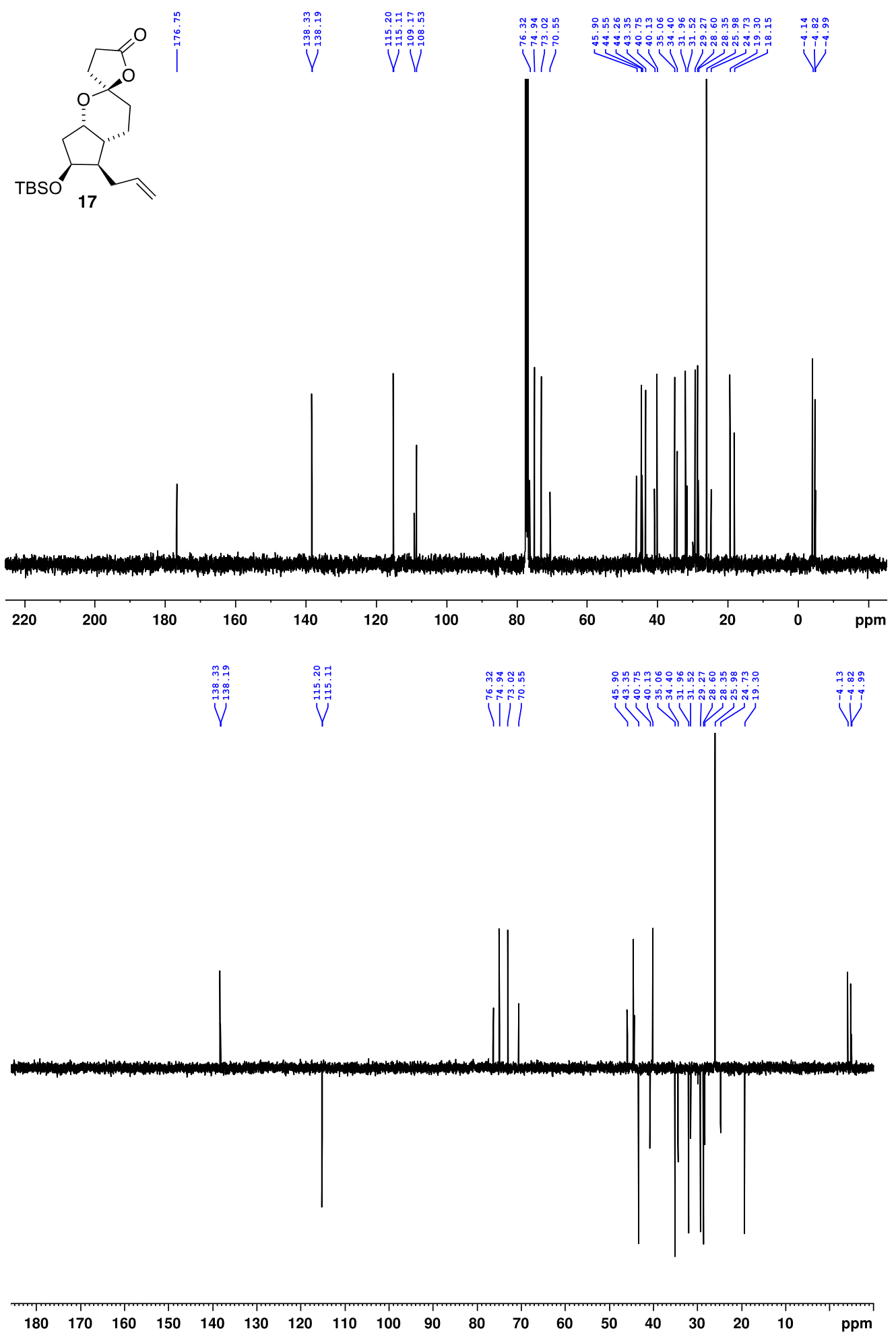

${ }^{13} \mathrm{C}$ NMR $\left(150 \mathrm{MHz}, \mathrm{CDCl}_{3}\right)$ and DEPT NMR $\left(150 \mathrm{MHz}, \mathrm{CDCl}_{3}\right)$ spectrum of 17 


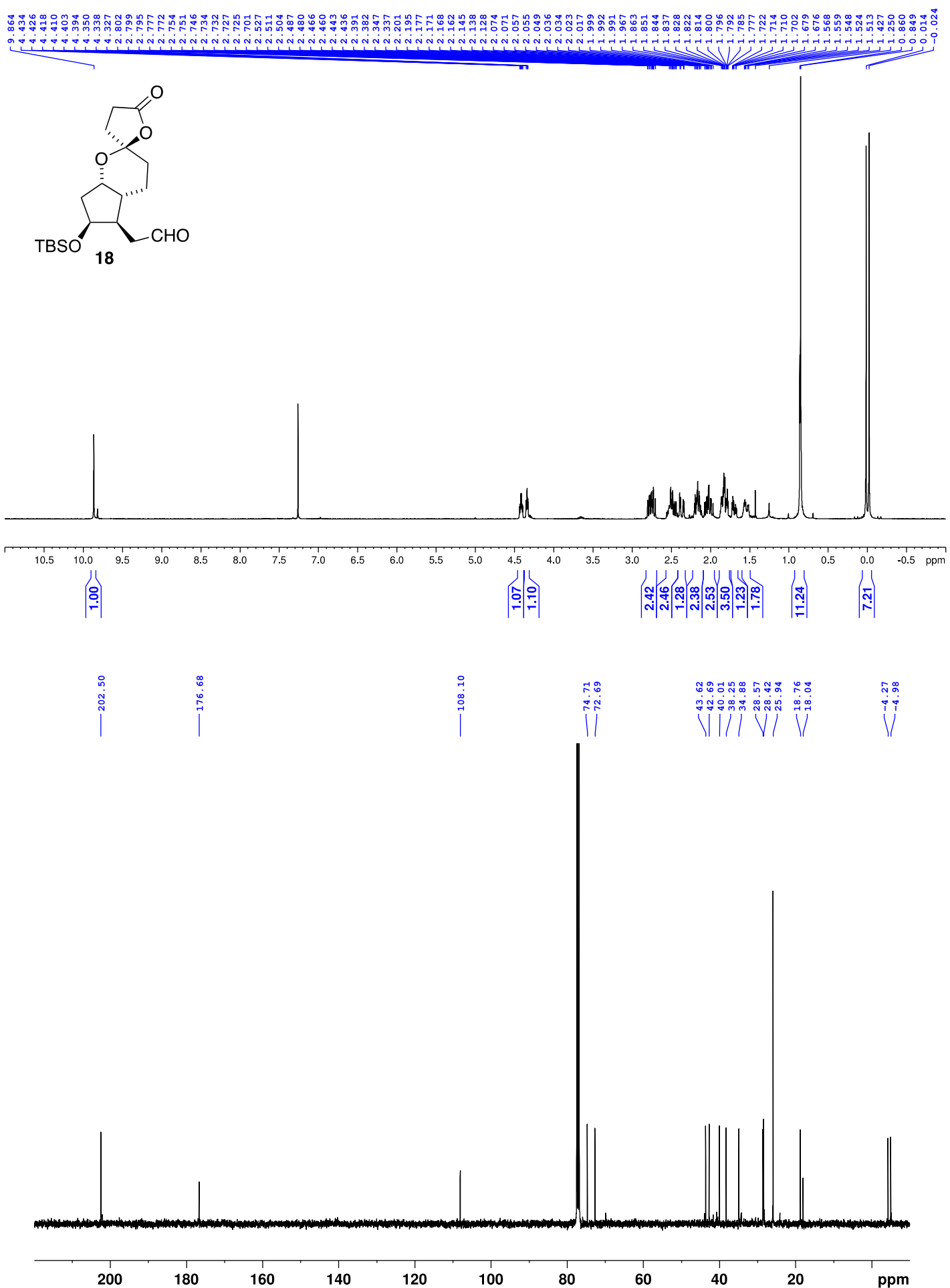

${ }^{1} \mathrm{H} \mathrm{NMR}\left(600 \mathrm{MHz}, \mathrm{CDCl}_{3}\right)$ and ${ }^{13} \mathrm{C} \mathrm{NMR}\left(150 \mathrm{MHz}, \mathrm{CDCl}_{3}\right)$ spectrum of 18 


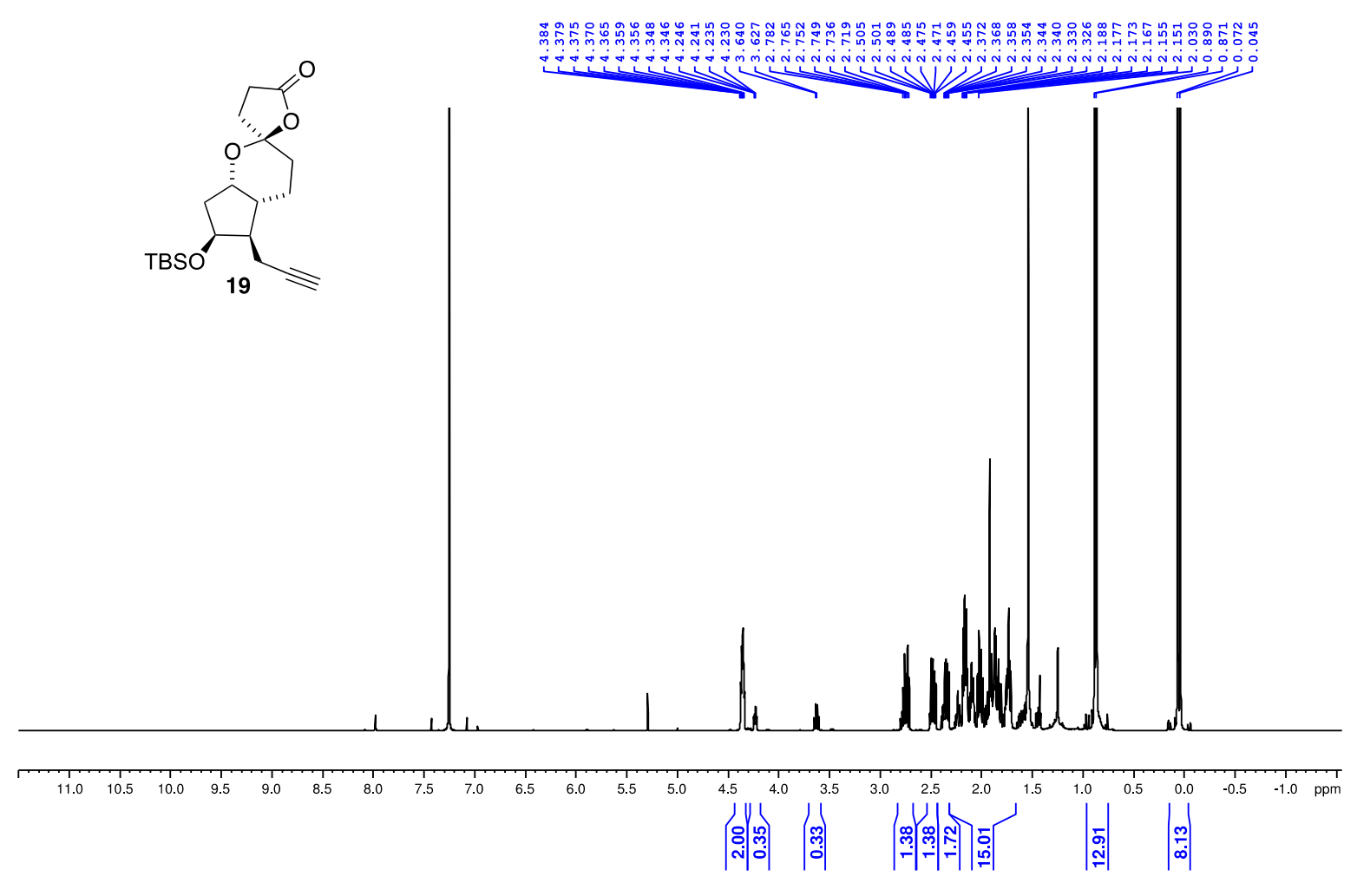

${ }^{1} \mathrm{H} \mathrm{NMR}\left(600 \mathrm{MHz}, \mathrm{CDCl}_{3}\right)$ spectrum of 19 

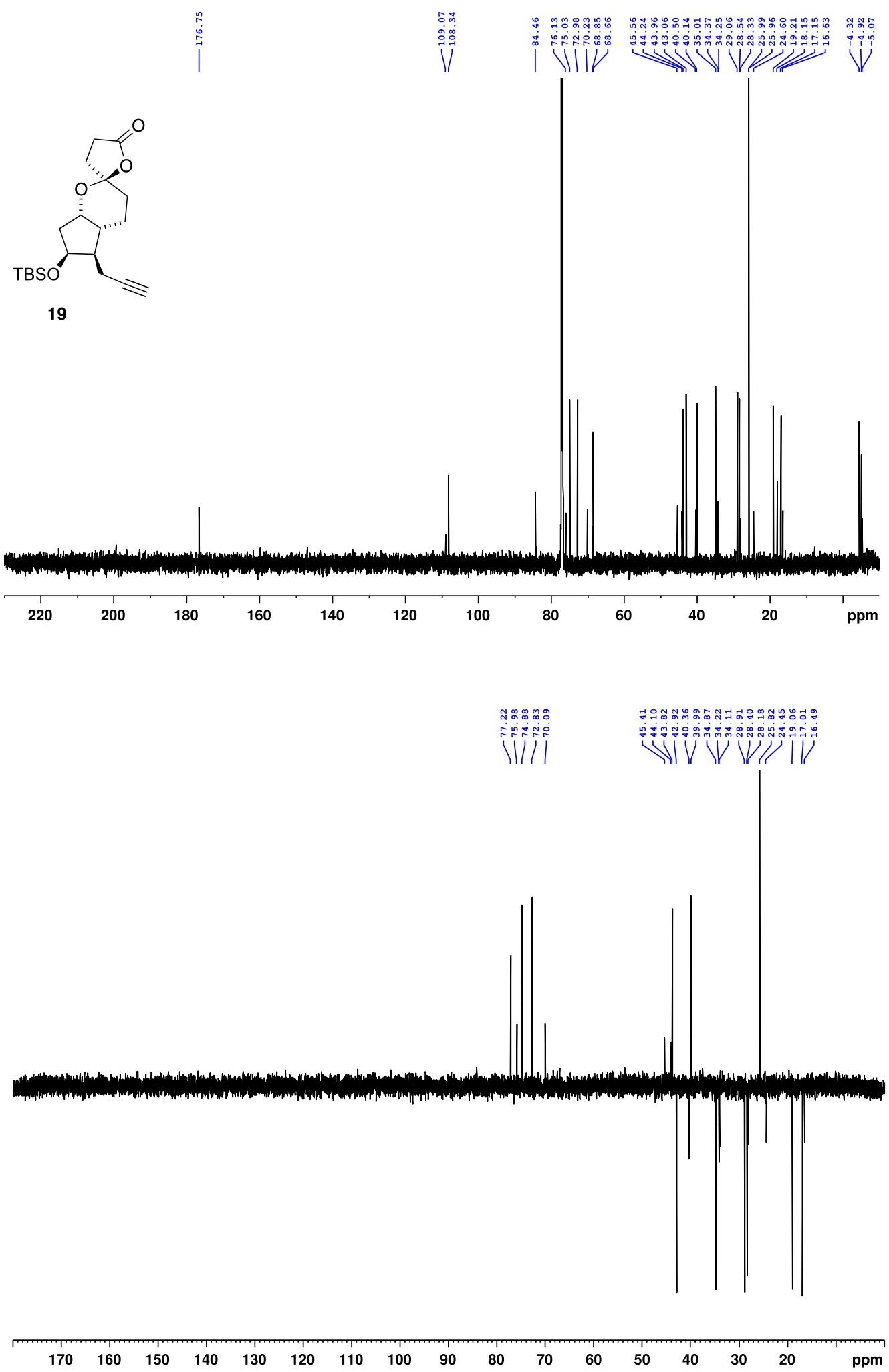

${ }^{13} \mathrm{C}$ NMR $\left(150 \mathrm{MHz}, \mathrm{CDCl}_{3}\right.$ )and DEPT NMR (150 MHz, CDCl${ }_{3}$ ) spectrum of 19 


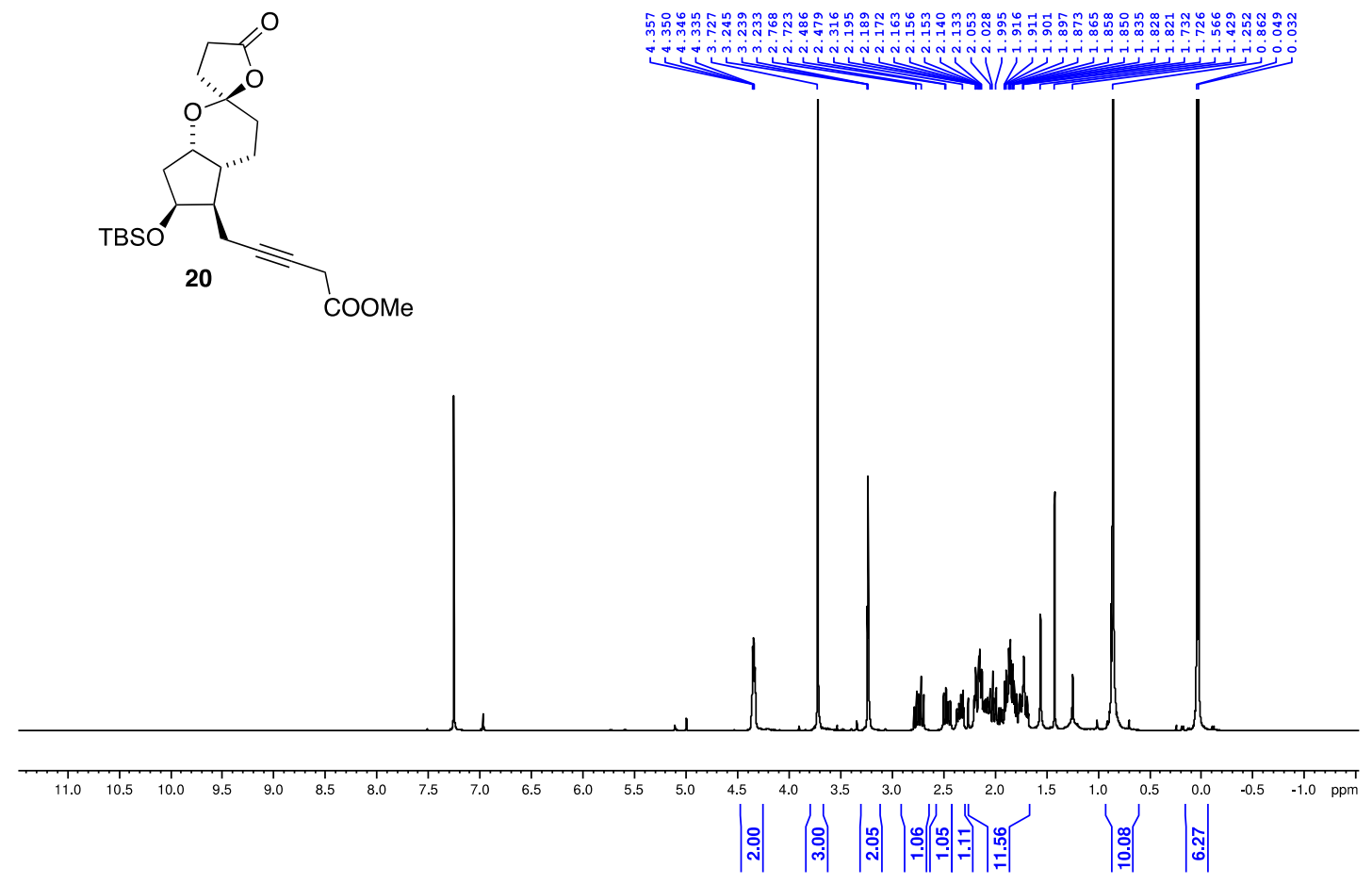

${ }^{1} \mathrm{H}$ NMR $\left(600 \mathrm{MHz}, \mathrm{CDCl}_{3}\right)$ spectrum of 20 

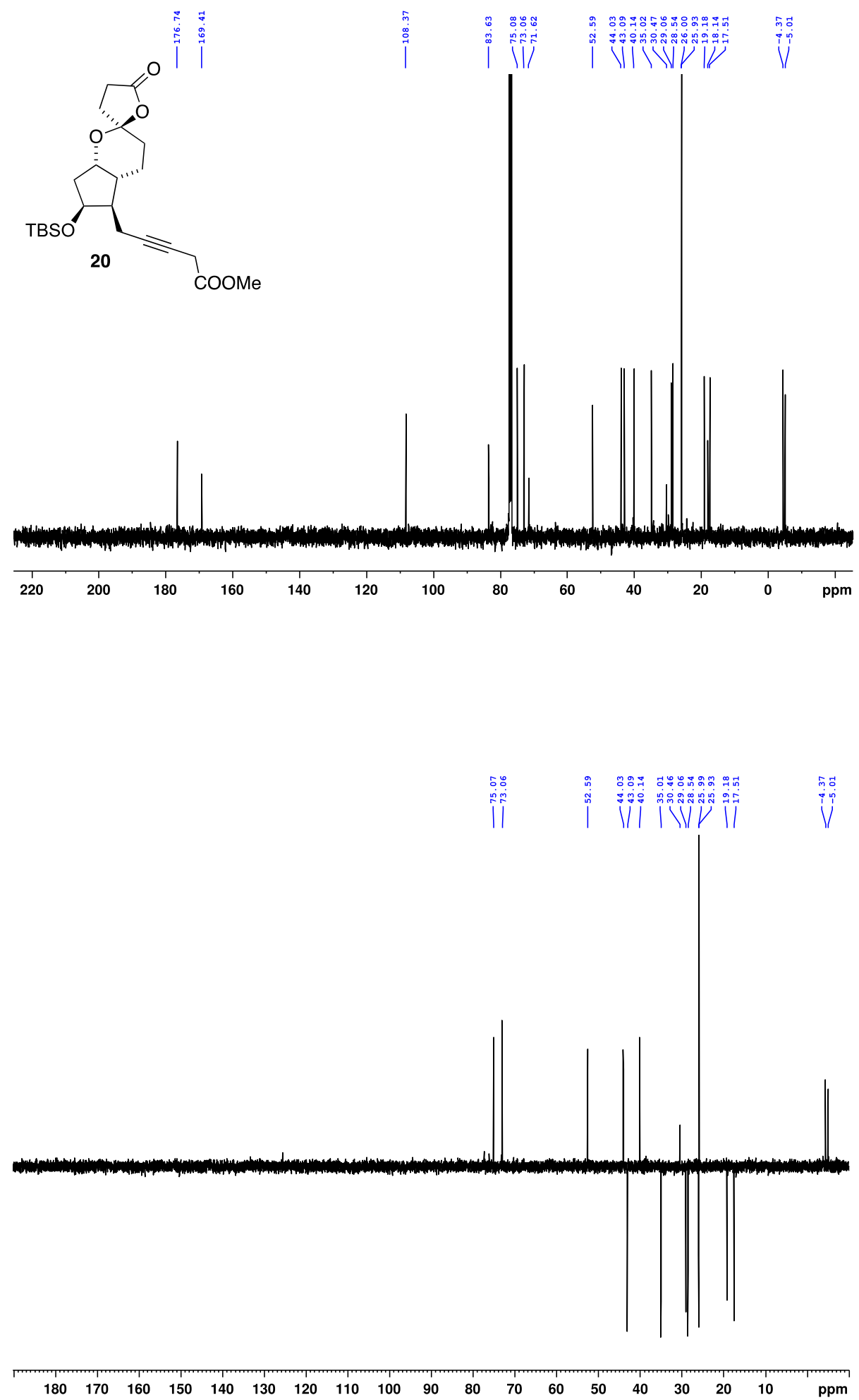

${ }^{13} \mathrm{C}$ NMR $\left(150 \mathrm{MHz}, \mathrm{CDCl}_{3}\right)$ and DEPT NMR $\left(150 \mathrm{MHz}, \mathrm{CDCl}_{3}\right)$ spectrum of 20 

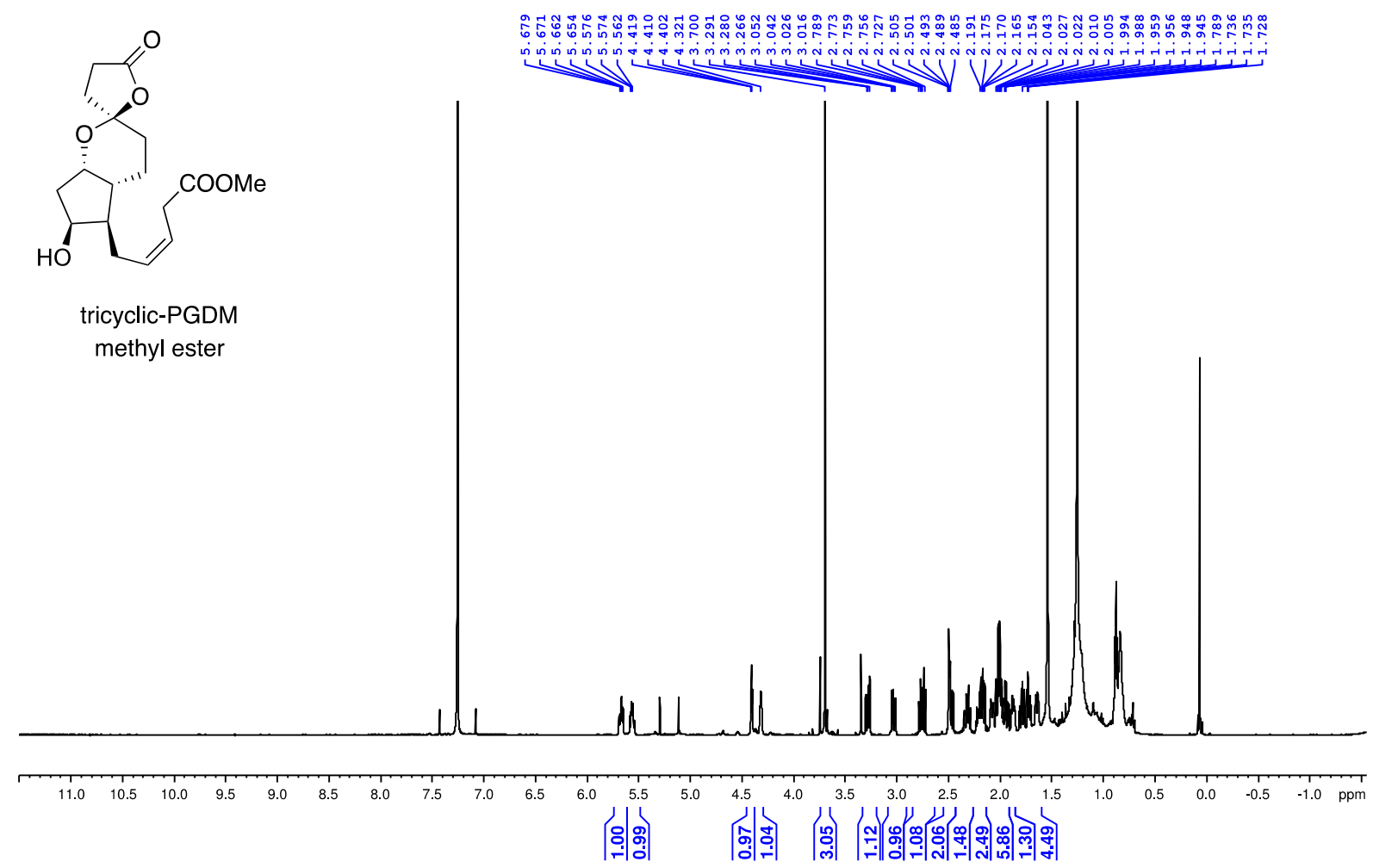

${ }^{1} \mathrm{H}$ NMR $\left(600 \mathrm{MHz}, \mathrm{CDCl}_{3}\right)$ spectrum of tricyclic-PGDM methyl ester 


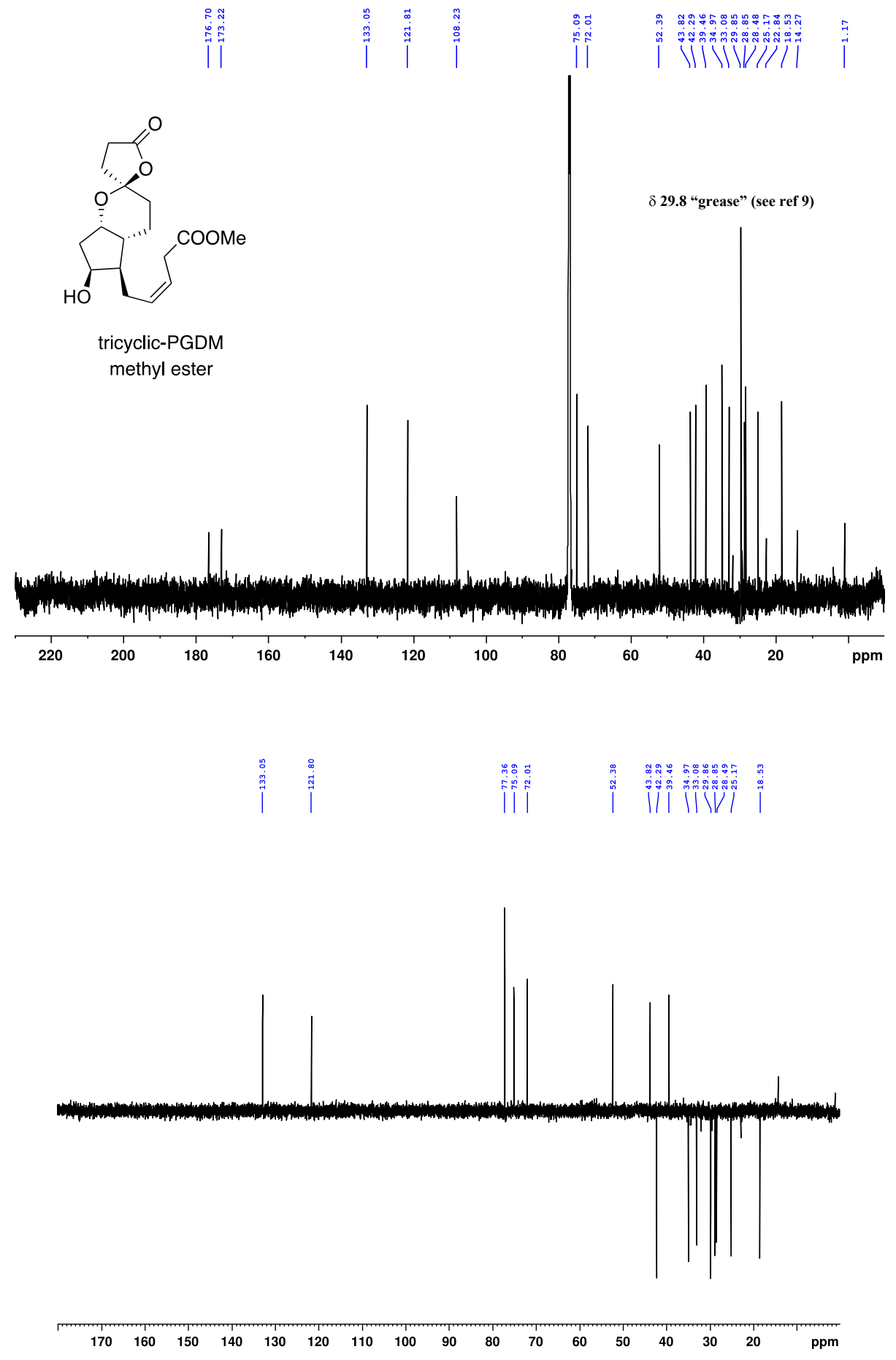

${ }^{13} \mathrm{C} \mathrm{NMR}\left(150 \mathrm{MHz}, \mathrm{CDCl}\right.$ ) and DEPT NMR (150 MHz, $\mathrm{CDCl}_{3}$ ) spectrum of tricyclic-PGDM methyl ester 


\section{References and Footnotes}

1. Deardorff, D. R.; Myles D. C.; MacFerrin, K. D. Tetrahedron Lett. 1985, 26, 5615-5618.

2. Tietze, L. F.; Stadler, C.; Bohnke, N.; Brasche, G.; Grube, A. Synlett 2007, 485-487.

3. Jeroncic, L. O.; Cabal, M. P.; Danishefsky, S. J.; Shulte, G. M. J. Org. Chem. 1991, 56, 387-395.

4. Yanagisawa, A.; Habaue, S.; Yasue, K.; Yamamoto, H. J. Am. Chem. Soc., 1994, 116, 6130-6141.

5. Guo, H.; O’Doherty, G. A. Org. Lett. 2005, 7, 3921-3924.

6. Marshall, J. A.; Liao, J. J. Org. Chem. 1998, 63, 5962-5970.

7. Myers, A. G.; Dragovich, P. S. J. Am. Chem. Soc. 1993, 115, 7021-7022.

8. Brown, D. G.; Velthuisen, E. J., Commerford, J. R.; Brisbois, R. G.; Hoye, T. R. J. Org. Chem. 1996, 61, 2540- 2541.

9. Gottlieb, H. E.; Kotlyar, V.; Nudelman, A. J. Org. Chem. 1997, 62, 7512-7515. 